\title{
Consequences of minimal seesaw with complex $\mu \tau$ antisymmetry of neutrinos
}

\author{
Rome Samanta, ${ }^{a}$ Probir Roy ${ }^{b}$ and Ambar Ghosal ${ }^{a}$ \\ ${ }^{a}$ Astroparticle Physics and Cosmology Division, Saha Institute of Nuclear Physics, HBNI, \\ Kolkata 700064, India \\ ${ }^{b}$ Center for Astroparticle Physics and Space Science, Bose Institute, \\ Kolkata 700091, India \\ E-mail: rome.samanta@saha.ac.in, probirrana@gmail.com, \\ ambar.ghosal@saha.ac.in
}

ABSTRACT: We propose a complex extension of $\mu \tau$ permutation antisymmetry in the neutrino Majorana matrix $M_{\nu}$. The latter can be realized for the Lagrangian by appropriate $\mathrm{CP}$ transformations on the neutrino fields. The resultant form of $M_{\nu}$ is shown to be simply related to that with a complex $(\mathrm{CP})$ extension of $\mu \tau$ permutation symmetry, with identical phenomenological consequences, though their group theoretic origins are quite different. We investigate those consequences in detail for the minimal seesaw induced by two strongly hierarchical right-chiral neutrinos $N_{1}$ and $N_{2}$ with the result that the Dirac phase is maximal while the two Majorana phases are either 0 or $\pi$. We further provide an uptodate discussion of the $\beta \beta 0 \nu$ process vis-a-vis ongoing and forthcoming experiments. Finally, a thorough treatment is given of baryogenesis via leptogenesis in this scenario, primarily with the assumption that the lepton asymmetry produced by the decays of $N_{1}$ only matters here with the asymmetry produced by $N_{2}$ being washed out. Tight upper and lower bounds on the mass of $N_{1}$ are obtained from the constraint of obtaining the correct observed range of the baryon asymmetry parameter and the role played by $N_{2}$ is elucidated thereafter. The mildly hierarchical right-chiral neutrino case (including the quasidegenerate possibility) is discussed in an appendix.

Keywords: Beyond Standard Model, CP violation, Neutrino Physics

ARXIV EPRINT: 1712.06555 


\section{Contents}

1 Introduction 1

2 Complex extension of $\mu \tau$ antisymmetry 4

3 Neutrino mixing angles and phases from $M_{\nu}^{\mathrm{CP} \mu A} \quad 6$

4 Origin of neutrino masses from a minimal seesaw $\quad 7$

$\begin{array}{llr}5 & \text { Neutrinoless double beta decay } & 8\end{array}$

$\begin{array}{llr}6 & \text { Baryogenesis via leptogenesis } & 9\end{array}$

$\begin{array}{lll}7 & \text { Numerical analysis: methodology and discussion } & 13\end{array}$

8 Concluding comments and discussion $\quad 21$

A Discussion of the case with mildly hierarchical RH neutrinos 22

\section{Introduction}

The masses and mixing properties [1] of the three light neutrinos continue to intrigue. We now know within reasonably precise ranges their two squared mass differences while from cosmology a fairly tight upper bound $[2,3]$ of $0.23 \mathrm{eV}$ has emerged on the sum of the three masses. The atmospheric mixing angle is now pinned around its maximal value of $\pi / 4$ and the solar mixing angle around the tri-bimaximal value while the reactor mixing angle is known to be significantly nonzero and close to $8^{0}$. The current trend of the data [4] suggests that the Dirac CP phase could be close to $3 \pi / 2$ but a definitive statement is yet to emerge. A specific prediction on the value of the latter will be very welcome. It is not known yet whether the light neutrinos are Dirac or Majorana in nature while relentless searches for the decisive neutrinoless double $\beta$-decay signal continue. For the latter case the two Majorana phases of the neutrinos also need to be predicted. Light Majorana neutrino masses can be generated by the seesaw mechanism [5-8] and a minimal version [9-21] with just two heavy right-chiral $(\mathrm{RH})$ neutrinos seems especially attractive. Further, the formulation of a viable scheme of baryogenesis via leptogenesis within this scenario is a challenging task. There has also been a substantial amount of work with discrete flavor symmetries of the light neutrino Majorana mass matrix: specifically real $\mu \tau$ permutation symmetry [22-31] and its complex (CP) extension [32-34] as well as real $\mu \tau$ permutation antisymmetry [35] but not the complex (CP) extension of that. This last mentioned topic will be the subject of our attention in this paper with the aim of predicting the neutrino $\mathrm{CP}$ phases. 
The neutrino mass terms in the Lagrangian density read

$$
-\mathcal{L}_{\text {mass }}^{\nu}=\frac{1}{2} \bar{\nu}_{L l}^{C}\left(M_{\nu}\right)_{l m} \nu_{L m}+\text { h.c. }
$$

with $\nu_{L l}^{C}=C{\overline{\nu_{L l}}}^{T}$ and the subscripts $l, m$ spanning the lepton flavor indices $e, \mu, \tau$ while the subscript $L$ denotes left-chiral neutrino fields. $M_{\nu}$ is a complex symmetric matrix $\left(M_{\nu}^{*} \neq M_{\nu}=M_{\nu}^{T}\right)$ in lepton flavor space. It can be put into a diagonal form by a similarity transformation with a unitary matrix $U$ :

$$
U^{T} M_{\nu} U=M_{\nu}^{d} \equiv \operatorname{diag}\left(\mathrm{m}_{1}, \mathrm{~m}_{2}, \mathrm{~m}_{3}\right) .
$$

Here $\mathrm{m}_{i}(i=1,2,3)$ are real and we assume that $m_{i} \geq 0$. We work in the basis in which charged leptons are mass diagonal. We are motivated by a flavor-based model constructed by Mohapatra and Nishi [42], which could accommodate a diagonal charged lepton mass matrix as well as a CP-transformed $\mu \tau$ interchange symmetry. Now we can relate $U$ to the $P M N S$ mixing matrix $U_{P M N S}$ :

$$
U=P_{\phi} U_{P M N S} \equiv P_{\phi}\left(\begin{array}{ccc}
c_{12} c_{13} & e^{i \frac{\alpha}{2}} s_{12} c_{13} & s_{13} e^{-i\left(\delta-\frac{\beta}{2}\right)} \\
-s_{12} c_{23}-c_{12} s_{23} s_{13} e^{i \delta} & e^{i \frac{\alpha}{2}}\left(c_{12} c_{23}-s_{12} s_{13} s_{23} e^{i \delta}\right) & c_{13} s_{23} e^{i \frac{\beta}{2}} \\
s_{12} s_{23}-c_{12} s_{13} c_{23} e^{i \delta} & e^{i \frac{\alpha}{2}}\left(-c_{12} s_{23}-s_{12} s_{13} c_{23} e^{i \delta}\right) & c_{13} c_{23} e^{i \frac{\beta}{2}}
\end{array}\right),
$$

where $P_{\phi}=\operatorname{diag}\left(e^{i \phi_{1}}, e^{i \phi_{2}} e^{i \phi_{3}}\right)$ is an unphysical diagonal phase matrix and $c_{i j} \equiv \cos \theta_{i j}$, $s_{i j} \equiv \sin \theta_{i j}$ with the mixing angles $\theta_{i j}=[0, \pi / 2]$. We work within the PDG convention [36] but denote our Majorana phases by $\alpha$ and $\beta$. CP-violation enters through nontrivial values of the Dirac phase $\delta$ and of the Majorana phases $\alpha, \beta$ with $\delta, \alpha, \beta=[0,2 \pi]$.

Real $\mu \tau$ symmetry [22-31] for $M_{\nu}$ implies that

$$
G^{T} M_{\nu} G=M_{\nu}
$$

where $G$ is a generator of a $\mathbb{Z}_{2}$ symmetry effecting $\mu \tau$ interchange. In the neutrino flavor space $G$ has the form

$$
G=\left(\begin{array}{lll}
1 & 0 & 0 \\
0 & 0 & 1 \\
0 & 1 & 0
\end{array}\right)
$$

A substantial amount of phenomenological work has been done following the consequences of (1.4). Additionally, its possible group theoretic origin from a more fundamental symmetry such as $A_{4}$ have been investigated [37-40]. However, this flavor symmetry leads to the prediction that $\theta_{13}=0$ which has now been excluded at more than $5.2 \sigma$ [41]. A way out was proposed [32-34] in terms of its complex $(\mathrm{CP})$ extension $\left(\mathrm{CP}^{\mu \tau}\right)$ with the postulate

$$
G^{T} M_{\nu} G=M_{\nu}^{*}
$$

The above can be realized as a Lagrangian symmetry by means of a CP transformation on the neutrino fields as

$$
\nu_{L l} \rightarrow i G_{l m} \gamma^{0} \nu_{L m}^{C}
$$


where $l, m$ are flavor indices and $\nu_{L m}^{C}=C \bar{\nu}_{L m}^{T}$. Detailed phenomenological consequences of (1.6) have been investigated in ref. [42].

Let us move on to real $\mu \tau$ permutation antisymmetry [35] which proposes that

$$
G^{T} M_{\nu} G=-M_{\nu}
$$

Note that the antisymmetry condition in (1.8) can written as a symmetry condition

$$
\mathcal{G}^{T} M_{\nu} \mathcal{G}=M_{\nu}
$$

where $\mathcal{G}=i G$. Now $\mathcal{G}$ is a generator of $\mathbb{Z}_{4}$ symmetry since $\mathcal{G}^{4}=1$. A sizable amount of work has earlier been done using $[43,44]$ the real $\mu \tau$ antisymmetry idea - including its application to the neutrino masses and mixing as well as its possible group theoretic origin from a more fundamental flavor symmetry such as $A_{5}$. However, the major phenomenological problem with exact real $\mu \tau$ antisymmetry is that it leads to a maximal solar neutrino mixing angle $\theta_{12}=\pi / 4$ as well as two degenerate light neutrinos - in conflict with experiment [45]. Perturbative modifications, in attempts to address these problems, unfortunately lead to a proliferation of extra unknown parameters. It is therefore highly desirable to propose an extension of this symmetry which is exact and therefore has the beauty of minimizing the number of input parameters. This is what we aim to do in this paper by proposing a complex $(\mathrm{CP})$ extension of $\mu \tau$ flavor antisymmetry $\mathrm{CP}^{\mu \tau A}$ and working out its various phenomenological implications. Complex extensions of $\mu \tau$ symmetry [32-34] and scaling symmetry [46-48] as well as their consequences have been worked out earlier. That is the direction of our thrust here for $\mu \tau$ antisymmetry.

We consider a complex $(\mathrm{CP})$ extension of $\mu \tau$ antisymmetry $\left(\mathrm{CP}^{\mu \tau A}\right)$ in the neutrino mass matrix. We show that this extension leads to a form of $M_{\nu}$ which is very simply related to that of $M_{\nu}$ for the $\mathrm{CP}^{\mu \tau}$ case. Moreover, this form allows neutrino mixing angles that are perfectly compatible with experiment both for a normal and for an inverted mass ordering. Additionally, specific statements can be made on $\mathrm{CP}$ violation in the neutrino sector. The Majorana phases $\alpha$ and $\beta$ have to be 0 or $\pi$ while Dirac CP violation has to be maximal with the phase $\delta$ being either $\pi / 2$ or $3 \pi / 2$. Further, reasonably nondegenerate values for the three neutrino masses can be generated by incorporating the minimal seesaw mechanism [9-21] implemented through two heavy right-chiral neutrinos $N_{R \ell}(\ell=1,2)$ with a $2 \times 2$ Majorana matrix $M_{R}$. (In case there is a third heavy Majorana neutrino, that is assumed to be much heavier and hence totally decoupled). Definitive predictions can be made on neutrinoless double beta decay for both types of mass ordering. Finally, a realistic scenario of baryogenesis via leptogenesis can be drawn and an acceptable value of the baryon asymmetry parameter $Y_{B}$ can be derived. Though the phenomenological consequences of $M_{\nu}^{\mathrm{CP}}{ }^{\mu \tau}$ and $M_{\nu}^{\mathrm{CP}^{\mu \tau A}}$ are identical, we feel that an uptodate detailed discussion of these along with some new results related to the scenario of baryogenesis via leptogenesis will be useful.

The new features in our work are (i) the demonstration that $M_{\nu}^{\mathrm{CP}^{\mu \tau A}}$ and $M_{\nu}^{\mathrm{CP}^{\mu \tau}}$ have identical phenomenological consequences despite there origin from different residual symmetries ( $Z_{4}$ and $Z_{2}$ respectively) and (ii) the use of the minimal seesaw with two heavy righthanded neutrinos (and consequently one massless left handed neutrino) to explore 
those consequences - in particular $\beta \beta 0 \nu$ decay and baryogenesis via leptogenesis. Let us highlight here what we propose to do in this paper. We plan to discuss the complex (CP) extension of $\mu \tau$ antisymmetry which has been analyzed so far in literature with its perturbative modifications only. Then we shall show how the resultant $M_{\nu}^{\mathrm{CP}^{\mu \tau A}}$ is simply related to $M_{\nu}^{\mathrm{CP}^{\mu \tau}}$ - the neutrino Majorana mass matrix from the complex (CP) extension of $\mu \tau$ symmetry — with identical phenomenological consequences despite the fact that their respective real components have almost entirely different predictions. We further emphasize the fact that $\mathrm{CP}^{\mu \tau}$ and $\mathrm{CP}^{\mu \tau A}$ are implemented with different residual symmetry generators, namely $\mathbb{Z}_{2}$ and $\mathbb{Z}_{4}$ respectively. Thus the corresponding high energy theory for these residual CP symmetries would likely be different. We then work out the consequences of $\mathrm{CP}^{\mu \tau A}$ in the framework of a minimal seesaw which leads to a vanishing value of one of the light neutrino masses and a very constraint range of the sum of the light neutrino masses as well. We also make an uptodate comparison of our conclusions on $\beta \beta 0 \nu$ decay with ongoing and forthcoming searches. We shall do a full parameter scan of the $3 \times 2$ Dirac mass matrix $m_{D}$ in the minimal seesaw scenario using the uptodate neutrino oscillation $3 \sigma$ global fit data. This in turn will lead us to perform a detailed computation related to the process baryogenesis via leptogenesis in our work which will result in new interesting upper and lower bounds on the mass of $N_{1}$. We shall also stress that these bounds could be erased if we consider a mildly hierarchical $\mathrm{RH}$ neutrino spectrum. We shall discuss the effect of $N_{2}$ on the final baryon asymmetry $Y_{B}$, in particular on the obtained upper and lower bounds on $M_{1}$ from the standard $N_{1}$ decay scenario.

The rest of the paper is organized as follows. In section 2 we explain the above mentioned complex extension $\mathrm{CP}^{\mu \tau A}$. section 3 contains a discussion of how the neutrino mixing angles and $\mathrm{CP}$ violating phases originate from $\mathrm{CP}^{\mu \tau A}$. In section 4 we discuss the origin of the neutrino masses from the minimal seesaw mechanism. The phenomenon of neutrinoless double beta decay is treated in section 5 . In section 6 we discuss baryogenesis via leptogenesis. Constraints on our model parameter space from all these phenomena are derived by numerical analysis in section 7 . The final section 8 contains a discussion of our conclusions. In an appendix we discuss what happens to our results if the right handed neutrinos are mildly hierarchical or quasidegenerate in mass.

\section{Complex extension of $\mu \tau$ antisymmetry}

We propose a complex extension of (1.8), namely

$$
G^{T} M_{\nu} G=-M_{\nu}^{*}, \mathcal{G}^{T} M_{\nu} \mathcal{G}=M_{\nu}^{*}
$$

The complex invariance condition in (2.1) can be obtained by the means of a CP transformation [49-65] on the neutrino fields as

$$
\nu_{L l} \rightarrow i \mathcal{G}_{l m} \gamma^{0} \nu_{L m}^{C}
$$

As we will see, since the real part of the resultant complex matrix exhibits $\mu \tau$ antisymmetry, we call the implemented CP symmetry as a complex extended $\mu \tau$ antisymmetry or simply 
complex $\mu \tau$ antisymmetry. This complex $\mu \tau$ antisymmetry $\mathrm{CP}^{\mu \tau A}$, generated by $\mathcal{G}$, needs to be broken in the charged lepton sector. Given that our charged lepton mass matrix $M_{\ell}$ is diagonal, a replacement of $M_{\nu}$ by $M_{\ell}$ in (2.1) would immediately lead to the unacceptable result $m_{\mu}=m_{\tau}$. There is an additional desirable reason for breaking $\mathrm{CP}^{\mu \tau A}$ in $M_{\ell}$. A nonzero Dirac CP violation is equivalent to

$$
\operatorname{Tr}\left[H_{\nu}, H_{\ell}\right]^{3} \neq 0,
$$

where the hermitian combinations are introduced as $H_{\nu}=M_{\nu}^{\dagger} M_{\nu}, H_{\ell}=M_{\ell}^{\dagger} M_{\ell}[66,67]$. A common $\mathrm{CP}$ symmetry $\mathcal{G}$ in both the sectors would imply

$$
\mathcal{G}^{T} H_{\nu}^{T} \mathcal{G}^{*}=H_{\nu}, \quad \mathcal{G}^{T} H_{\ell}^{T} \mathcal{G}^{*}=H_{\ell}
$$

From (2.4) it follows that $\operatorname{Tr}\left[H_{\nu}, H_{\ell}\right]^{3}=0$ which leads to $\sin \delta=0$ i.e. a vanishing Dirac $\mathrm{CP}$ violation. Though this is still a possibility, it goes against the current trend of the data [4]. The most general structure of $M_{\nu}$ that satisfies the $\mathrm{CP}^{\mu \tau A}$ condition (2.1) can be worked out to be

$$
M_{\nu}^{\mathrm{CP}^{\mu \tau A}}=\left(\begin{array}{ccc}
i A & B & -B^{*} \\
B & C & i D \\
-B^{*} & i D & -C^{*}
\end{array}\right),
$$

where $A, D$ are real and $B, C$ are complex mass dimensional quantities which are a priori unknown. The matrix $M_{\nu}^{\mathrm{CP}}{ }^{\mu \tau A}$ can also be written as

$$
M_{\nu}^{\mathrm{CP}}{ }^{\mu \tau A}=\left(\begin{array}{ccc}
0 & B_{1} & -B_{1} \\
B_{1} & C_{1} & 0 \\
-B_{1} & 0 & -C_{1}
\end{array}\right)+i\left(\begin{array}{ccc}
A & B_{2} & B_{2} \\
B_{2} & C_{2} & D \\
B_{2} & D & C_{2}
\end{array}\right)
$$

where $B=B_{1}+i B_{2}$ and $C=C_{1}+i C_{2}$ with $B_{1,2}$ and $C_{1,2}$ being real. Note that the real part of the matrix in (2.6) is invariant under $\mu \tau$ antisymmetry while the imaginary part is $\mu \tau$ symmetric. Thus the entire source of corrections here to real $\mu \tau$ antisymmetry arises from the imaginary $\mu \tau$ symmetric part.

Here we make the interesting observation that $i M_{\nu}^{\mathrm{CP}^{\mu \tau A}}$ yields a neutrino Majorana mass matrix that is complex $\mu \tau$ symmetric since

$$
i M_{\nu}^{\mathrm{CP}^{\mu \tau A}}=\left(\begin{array}{ccc}
-A & i B & -i B^{*} \\
i B & i C & -D \\
-i B^{*} & -D & -i C^{*}
\end{array}\right) \equiv M_{\nu}^{\mathrm{CP}^{\mu \tau}}
$$

This is since

$$
G^{T}\left(i M_{\nu}^{\mathrm{CP}^{\mu \tau A}}\right) G=\left(i M_{\nu}^{\mathrm{CP}}{ }^{\mu \tau A}\right)^{*}
$$

Therefore the phenomenological consequences of a complex (CP) $\mu \tau$ symmetric form of $M_{\nu}$ and a complex antisymmetric form of the same would be identical. Nevertheless, we deem it worthwhile to give a detailed updated discussion of its phenomenological consequences and highlight some new effects such as the role of another heavy $\mathrm{RH}$ neutrino $\mathrm{N}_{2}$ on the process of baryogenesis via leptogenesis in a standard $N_{1}$-leptogenesis scenario. 


\section{Neutrino mixing angles and phases from $M_{\nu}^{\mathrm{CP}}$}

Eqs. (1.2) and (2.1) together imply [32-34] that

$$
\mathcal{G} U^{*}=U \tilde{d}
$$

where

$$
\tilde{d}_{l m}= \pm \delta_{l m}
$$

Let us take

$$
\tilde{d}=\operatorname{diag}\left(\tilde{\mathrm{d}_{1}}, \tilde{\mathrm{d}}_{2}, \tilde{\mathrm{d}}_{3}\right)
$$

where each $\tilde{d}_{i}(i=1,2,3)$ can be +1 or -1 . Eq. (3.1) can explicitly be written, by taking $\mathcal{G}$ equal to $i$ times $G$ as given in (1.5), namely

$$
\left(\begin{array}{ccc}
i U_{e 1}^{*} & i U_{e 2}^{*} & i U_{e 3}^{*} \\
i U_{\tau 1}^{*} & i U_{\tau 2}^{*} & i U_{\tau 3}^{*} \\
i U_{\mu 1}^{*} & i U_{\mu 2}^{*} & i U_{\mu 3}^{*}
\end{array}\right)=\left(\begin{array}{ccc}
\tilde{d}_{1} U_{e 1} & \tilde{d}_{2} U_{e 2} & \tilde{d}_{3} U_{e 3} \\
\tilde{d}_{1} U_{\mu 1} & \tilde{d}_{2} U_{\mu 2} & \tilde{d}_{3} U_{\mu 3} \\
\tilde{d}_{1} U_{\tau 1} & \tilde{d}_{2} U_{\tau 2} & \tilde{d}_{3} U_{\tau 3}
\end{array}\right)
$$

which is equivalent to six independent equations:

$$
\begin{aligned}
& i U_{e 1}^{*}=\tilde{d}_{1} U_{e 1}, i U_{e 2}^{*}=\tilde{d}_{2} U_{e 2}, i U_{e 3}^{*}=\tilde{d}_{3} U_{e 3} . \\
& i U_{\tau 1}^{*}=\tilde{d}_{1} U_{\mu 1}, i U_{\tau 2}^{*}=\tilde{d}_{2} U_{\mu 2}, i U_{\tau 3}^{*}=\tilde{d}_{3} U_{\mu 3} .
\end{aligned}
$$

In order to calculate the Majorana phases in a way that avoids the unphysical phases, it is useful to construct two rephasing invariants [68-71]

$$
\mathcal{I}_{1}=U_{e 1} U_{e 2}^{*}, \mathcal{I}_{2}=U_{e 1} U_{e 3}^{*} .
$$

By using (3.5), $\mathcal{I}_{1,2}$ can be written as free of the unphysical phases, namely

$$
\mathcal{I}_{1}=\tilde{d}_{1} \tilde{d}_{2} U_{e 1}^{*} U_{e 2}, \mathcal{I}_{2}=\tilde{d}_{1} \tilde{d}_{3} U_{e 1}^{*} U_{e 3} .
$$

After equating the two different expressions for $\mathcal{I}_{1,2}$ in (3.7) and (3.8), we obtain

$$
\begin{aligned}
& \mathcal{I}_{1}=c_{12} s_{12} c_{13}^{2} e^{-i \frac{\alpha}{2}}=\tilde{d}_{1} \tilde{d}_{2} c_{12} s_{12} c_{13}^{2} e^{i \frac{\alpha}{2}}, \\
& \mathcal{I}_{2}=c_{12} s_{13} c_{13} e^{i\left(\delta-\frac{\beta}{2}\right)}=\tilde{d}_{1} \tilde{d}_{3} c_{12} s_{13} c_{13} e^{-i\left(\delta-\frac{\beta}{2}\right)} .
\end{aligned}
$$

Eqs. (3.9) and (3.10) imply

$$
e^{i \alpha}=\tilde{d}_{1} \tilde{d}_{2}, e^{2 i(\delta-\beta / 2)}=\tilde{d}_{1} \tilde{d}_{3}
$$

Thus

$$
\begin{aligned}
& \tilde{d}_{1} \tilde{d}_{2}=+1 \Rightarrow \alpha=0, \quad \tilde{d}_{1} \tilde{d}_{2}=-1 \Rightarrow \alpha=\pi, \\
& \tilde{d}_{1} \tilde{d}_{3}=+1 \Rightarrow \delta-\frac{\beta}{2}=0, \quad \tilde{d}_{1} \tilde{d}_{3}=-1 \Rightarrow \delta-\frac{\beta}{2}=\pi / 2 .
\end{aligned}
$$




\begin{tabular}{|c|c|c|c|c|c|c|c|}
\hline$\tilde{d}_{1}$ & $\tilde{d}_{2}$ & $\tilde{d}_{3}$ & $\tilde{d}_{1} \tilde{d}_{2}$ & $\tilde{d}_{1} \tilde{d}_{3}$ & $\alpha$ & $\beta$ & $\cos \delta$ \\
\hline 1 & 1 & 1 & 1 & 1 & 0 & $\pi$ & 0 \\
1 & -1 & 1 & -1 & 1 & $\pi$ & $\pi$ & 0 \\
1 & 1 & -1 & 1 & -1 & 0 & 0 & 0 \\
1 & -1 & -1 & -1 & -1 & $\pi$ & 0 & 0 \\
\hline
\end{tabular}

Table 1. Predictions on the CP phases.

Taking the modulus squared of the third equality in (3.6), namely $\left|U_{\tau 3}\right|=\left|U_{\mu 3}\right|$, we obtain

$$
c_{23}^{2}=s_{23}^{2}
$$

which implies $\theta_{23}=\pi / 4$, i.e. a maximal atmospheric mixing. Incorporating this last result, the modulus square of the first or the second equality in (3.6) leads after some algebra to the relation

$$
2 c_{12} s_{12} c_{13} s_{13} \cos \delta=0 .
$$

Given the experimentally observed nonvanishing values for all the mixing angles, (3.15) leads to a maximal Dirac CP-violation

$$
\cos \delta=0 \text { i.e. } \delta=\pi / 2 \text { or } 3 \pi / 2 \text {. }
$$

It then follows from (3.13) that

$$
\tilde{d}_{1} \tilde{d}_{3}=+1 \Rightarrow \beta=\pi, \tilde{d}_{1} \tilde{d}_{3}=-1 \Rightarrow \beta=0 .
$$

We can summarize our results on $\alpha, \beta$ and $\cos \delta$ in table 1 .

\section{Origin of neutrino masses from a minimal seesaw}

We now discuss the realization of the complex extended $\mu \tau$ antisymmetric mass matrix $M_{\nu}^{\mathrm{CP}}{ }^{\mu \tau A}$ through the minimal seesaw mechanism [9-21] mentioned earlier. This mechanism makes use of two heavy right chiral neutrino fields $N_{R i}(i=1,2)$ with a Majorana mass matrix $M_{R}$. We work in a basis in which $M_{R}$ is real, positive and diagonal [72, 73], i.e., $M_{R}=\operatorname{diag}\left(\mathrm{M}_{1}, \mathrm{M}_{2}\right), M_{1,2}>0$. With $m_{D}$ as the Dirac mass matrix, the neutrino mass terms read

$$
-\mathcal{L}_{\text {mass }}^{\nu, N}=\bar{N}_{R i}\left(m_{D}\right)_{i \alpha} l_{L \alpha}+\frac{1}{2} \bar{N}_{R i}\left(M_{R}\right)_{i j} \delta_{i j} N_{R j}^{C}+\text { h.c. },
$$

where $l_{L \alpha}=\left(\nu_{L \alpha} e_{L \alpha}\right)^{T}$ is the SM lepton doublet of flavor $\alpha$. The effective light neutrino mass matrix is given by the standard seesaw relation

$$
M_{\nu}=-m_{D}^{T} M_{R}^{-1} m_{D}
$$

In this case (2.1) is satisfied through the symmetry transformation on $m_{D}$ as

$$
m_{D} \mathcal{G}=-i m_{D}^{*}
$$


so long as $M_{R}^{-1}$ is real. The most general form of $m_{D}$ that satisfies (4.3) can be parametrized as

$$
m_{D}=\left(\begin{array}{lll}
\sqrt{2} a_{1} e^{i \pi / 4} & b_{1} e^{i \theta_{1}} & i b_{1} e^{-i \theta_{1}} \\
\sqrt{2} a_{2} e^{i \pi / 4} & b_{2} e^{i \theta_{2}} & i b_{2} e^{-i \theta_{2}}
\end{array}\right),
$$

where the parameters $a_{1,2}, b_{1,2}$ and $\theta_{1,2}$ are real.

The form of the effective light neutrino mass matrix $M_{\nu}$ that now emerges is given below:

$$
\begin{aligned}
& M_{\nu}^{\mathrm{CP}^{\mu \tau A}}= \\
& \left(\begin{array}{ccc}
-2 i\left(x_{1}^{2}+x_{2}^{2}\right) & -\sqrt{2} e^{i \pi / 4}\left(x_{1} y_{1} e^{i \theta_{1}}+x_{2} y_{2} e^{i \theta_{2}}\right) & -i \sqrt{2} e^{i \pi / 4}\left(x_{1} y_{1} e^{-i \theta_{1}}+x_{2} y_{2} e^{-i \theta_{2}}\right) \\
-\sqrt{2} e^{i \pi / 4}\left(x_{1} y_{1} e^{i \theta_{1}}+x_{2} y_{2} e^{i \theta_{2}}\right) & -\left(e^{2 i \theta_{1}} y_{1}^{2}+e^{2 i \theta_{2}} y_{2}^{2}\right) & -i\left(y_{1}^{2}+y_{2}^{2}\right) \\
-i \sqrt{2} e^{i \pi / 4}\left(x_{1} y_{1} e^{-i \theta_{1}}+x_{2} y_{2} e^{-i \theta_{2}}\right) & -i\left(y_{1}^{2}+y_{2}^{2}\right) & e^{-2 i \theta_{1}} y_{1}^{2}+e^{-2 i \theta_{2}} y_{2}^{2}
\end{array}\right) .
\end{aligned}
$$

In (4.5) we have introduced new real parameters $x_{1,2}$ and $y_{1,2}$ which are obtained by scaling $a_{1,2}$ and $b_{1,2}$ with the square roots of the respective $R H$ neutrino masses $M_{1,2}$, i.e.

$$
\frac{a_{1,2}}{\sqrt{M_{1,2}}}=x_{1,2}, \frac{b_{1,2}}{\sqrt{M_{1,2}}}=y_{1,2} .
$$

The lightest neutrino mass, either $m_{1}$ for a normal mass ordering or $m_{3}$ for an inverted mass ordering, has to vanish since $\operatorname{det} M_{\nu}^{\mathrm{CP}^{\mu \tau A}}=0$. Furthermore, one of the phases of $M_{\nu}$ (say $\theta_{1}$ ) can be rotated by the phase matrix $P_{\phi}=\operatorname{diag}\left(1, e^{i \phi}, e^{-i \phi}\right.$ ) with the choice $\theta_{1}=-\phi$. Thus we are left with only the phase difference $\theta_{2}-\theta_{1}$ in $M_{\nu}$. We can now rename $\theta_{2}-\theta_{1}$ as $\theta$. Without loss of generality, this is also equivalent to the choice $\theta_{1}=0$ and $\theta_{2}=\theta$ in $m_{D}$. From now on we shall use this redefined phase $\theta$ for both $M_{\nu}$ and $m_{D}$.

\section{Neutrinoless double beta decay}

The rare $\beta \beta 0 \nu$ process can arise from the following decay of a nucleus

$$
(A, Z) \longrightarrow(A, Z+2)+2 e^{-} .
$$

In (5.1) lepton number is violated by two units. Unlike in neutrinoful double $\beta$-decay, which is a sequence of two single $\beta$-decays, final state neutrinos are absent in the $\beta \beta 0 \nu$ process. The latter can go through via an appropriate neutrino loop only if the light neutrinos have Majorana masses. Therefore any observation of such a decay will unambiguously establish the Majorana nature of the light neutrinos. The half-life, corresponding to $\beta \beta 0 \nu$ decay, can be expressed as

$$
\frac{1}{T_{1 / 2}^{0 \nu}}=G\left|M_{e e}\right|^{2}|\mathcal{M}|^{2} m_{e}^{-2}
$$

Here $G$ is the two-body phase space factor and $M_{e e}$ is the $(1,1)$ element of the effective light neutrino mass matrix $M_{\nu}$, cf. (1.1). Moreover, $\mathcal{M}$ is the nuclear matrix element (NME) and $m_{e}$ is the electron mass. $M_{e e}$ can be written within our convention as

$$
M_{e e}=c_{12}^{2} c_{13}^{2} m_{1}+s_{12}^{2} c_{13}^{2} m_{2} e^{i \alpha}+s_{13}^{2} m_{3} e^{i(\beta-2 \delta)} .
$$


Significant upper limits on $\left|M_{e e}\right|$ are available from ongoing search experiments for $\beta \beta 0 \nu$ decay. KamLAND-Zen [74] and EXO [75] had earlier constrained this value to be $<0.35 \mathrm{eV}$. But the most impressive upper bound till date is provided by GERDA phaseII data [76]: $M_{e e}<0.098 \mathrm{eV}$. As explained in section 3, we have four sets of values for the three $\mathrm{CP}$ violating phases $\alpha, \beta, \delta$ in the neutrino sector corresponding to the four independent $\tilde{d}$ matrices. Furthermore, we need to consider both kinds of light neutrino mass ordering: normal and inverted. Thus we shall have eight sets of predictions for $\left|M_{e e}\right|$ from our modelled $M_{\nu}$. These will be detailed in our section on numerical analysis.

At this stage it may be useful to point out how (5.3) simplifies in our model for the specific cases of normal and inverted mass ordering subject to the condition given in eq. (3.16). For a normal mass ordering, we have $m_{1}=0$ and further

$$
\begin{aligned}
& \alpha=0, \beta=0 ; \alpha=\pi, \beta=\pi:\left|M_{e e}\right|=\left(s_{12}^{4} c_{13}^{4} m_{2}^{2}+s_{13}^{4} m_{3}^{2}-2 s_{12}^{2} s_{13}^{2} c_{13}^{2} m_{2} m_{3}\right)^{1 / 2} \text { (Normal), } \\
& \alpha=0, \beta=\pi ; \alpha=\pi, \beta=0:\left|M_{e e}\right|=\left(s_{12}^{4} c_{13}^{4} m_{2}^{2}+s_{13}^{4} m_{3}^{2}+2 s_{12}^{2} s_{13}^{2} c_{13}^{2} m_{2} m_{3}\right)^{1 / 2} \text { (Normal). }
\end{aligned}
$$

Note that the value of $\left|M_{e e}\right|$ becomes somewhat less here since the terms involving $m_{3}$ are suppressed by the powers of $s_{13}$. For an inverted mass ordering, $m_{3}=0$ and $\left|M_{e e}\right|$ becomes independent of $\beta$ and $\delta$. Indeed, we have

$$
\begin{aligned}
& \alpha=0:\left|M_{e e}\right|=c_{13}^{2}\left(c_{12}^{4} m_{1}^{2}+s_{12}^{4} m_{2}^{2}+2 c_{12}^{2} s_{12}^{2} m_{1} m_{2}\right)^{1 / 2} \text { (Inverted), } \\
& \alpha=\pi:\left|M_{e e}\right|=c_{13}^{2}\left(c_{12}^{4} m_{1}^{2}+s_{12}^{4} m_{2}^{2}-2 c_{12}^{2} s_{12}^{2} m_{1} m_{2}\right)^{1 / 2} \text { (Inverted). }
\end{aligned}
$$

Since $\Delta m_{21}^{2} \ll\left|\Delta m_{32}^{2}\right|$, in this case we can assume $m_{1} \approx m_{2} \approx \sqrt{\left|\Delta m_{32}^{2}\right|}$. Thus, for the two allowed values of $\alpha$, we have

$$
\begin{aligned}
& \alpha=0:\left|M_{e e}\right| \simeq \sqrt{\left|\Delta m_{32}^{2}\right|} c_{13}^{2} \text { (Inverted), } \\
& \alpha=\pi:\left|M_{e e}\right| \simeq \sqrt{\left|\Delta m_{32}^{2}\right|} c_{13}^{2}\left[\left\{1-2 s_{12}^{2}\right\}^{2}\right] \text { (Inverted). }
\end{aligned}
$$

We see that $\left|M_{e e}\right|$ for $\alpha=\pi$ is suppressed here relative to its value in the $\alpha=0$ case.

\section{Baryogenesis via leptogenesis}

To start with, we recall the observed range of $Y_{B}=\left(n_{B}-n_{\bar{B}}\right) / s$ - the ratio of baryonic minus antibaryonic number density to the entropy density - namely

$$
8.55 \times 10^{-11}<Y_{B}<8.77 \times 10^{-11} .
$$

$\mathrm{CP}$ violating decays from heavy Majorana neutrinos that are out of equilibrium generate a lepton asymmetry [77-79]. The latter is later converted into a baryon asymmetry by sphaleron transitions [80]. The appropriate part of the Lagrangian for the process can be written as

$$
-\mathcal{L}=\lambda_{i \alpha} \bar{N}_{R i} \tilde{\phi}^{\dagger} l_{L \alpha}+\frac{1}{2} \bar{N}_{R i}\left(M_{R}\right)_{i j} \delta_{i j} N_{R j}^{C}+\text { h.c. },
$$


where $\tilde{\phi}=i \tau_{2} \phi^{*}$, with $\phi=\left(\phi^{+} \phi^{0}\right)^{T}$ being the Higgs doublet. The possible decays of $N_{i}$ from (6.2) are $N_{i} \rightarrow e_{\alpha}^{-} \phi^{+}, \nu_{\alpha} \phi^{0}, e_{\alpha}^{+} \phi^{-}$, and $\nu_{\alpha}^{C} \phi^{0 *}$. The CP asymmetry parameter $\varepsilon_{i}^{\alpha}$, that is a measure of the required $\mathrm{CP}$ violation, arises from the interference between the tree level, one loop self energy and one loop vertex diagrams [77] for the decay of $N_{i}$. It has the general expression [81]

$$
\varepsilon_{i}^{\alpha}=\frac{1}{4 \pi v^{2} h_{i i}} \sum_{j \neq i}\left\{\operatorname{Im}\left[h_{i j}\left(m_{D}\right)_{i \alpha}\left(m_{D}^{*}\right)_{j \alpha}\right] g\left(x_{i j}\right)+\frac{\operatorname{Im}\left[h_{j i}\left(m_{D}\right)_{i \alpha}\left(m_{D}^{*}\right)_{j \alpha}\right]}{1-x_{i j}}\right\}
$$

where $h_{i j} \equiv\left(m_{D} m_{D}^{\dagger}\right)_{i j},\left\langle\phi^{0}\right\rangle=v / \sqrt{2}$ (so that $m_{D}=v \lambda / \sqrt{2}$ ) and $x_{i j}=M_{j}^{2} / M_{i}^{2}$. In addition, the loop function $g\left(x_{i j}\right)$ has the standard expression

$$
g\left(x_{i j}\right)=\frac{\sqrt{x_{i j}}}{1-x_{i j}}+f\left(x_{i j}\right)
$$

with

$$
f\left(x_{i j}\right)=\sqrt{x_{i j}}\left[1-\left(1+x_{i j}\right) \ln \left(\frac{1+x_{i j}}{x_{i j}}\right)\right] .
$$

Before proceeding further in the calculation of $\varepsilon_{i}^{\alpha}$ in our scenario, we need to address some important issues related to leptogenesis. For hierarchical $\mathrm{RH}$ neutrino masses $M_{2} \gg M_{1}$ (some discussion of the mildly hierarchical RH neutrino case including quasidegenerate masses is given later in the appendix), it can be shown that only the decays of $N_{1}$ matter for the creation of lepton asymmetry while the latter created from the heavier neutrinos gets washed out [82] significantly. Therefore, in general, only $\varepsilon_{1}^{\alpha}$ is the pertinent quantity in a hierarchical leptogenesis scenario. Nevertheless, there are certain circumstances in which the decays of $N_{2,3}$ do affect the final baryon asymmetry [87-89]. Furthermore, flavor plays an important role in the phenomenon of leptogenesis [83-86]. Assuming the temperature scale of the process to be $T \sim M_{1}$, the rates of the charged lepton Yukawa interaction categorize leptogenesis into the following three categories.

1) Unflavored leptogenesis: $T \sim M_{1}>10^{12} \mathrm{GeV}$, when all interactions with all flavors are out of equilibrium: in this case all the flavors are indistinguishable; therefore the total $\mathrm{CP}$ asymmetry is a sum over all flavors, i.e. $\varepsilon_{1}=\sum_{\alpha} \varepsilon_{1}^{\alpha}$ and the final baryon asymmetry $Y_{B}$ is proportional to $\varepsilon_{1}$.

2) $\tau$-flavored leptogenesis: $10^{9} \mathrm{GeV}<T \sim M_{1}<10^{12} \mathrm{GeV}$, when only the $\tau$ flavor is in equilibrium and hence distinguishable. In this regime there are two pertinent $\mathrm{CP}$ asymmetry parameters; $\varepsilon_{1}^{\tau}$ and $\varepsilon_{1}^{(2)}=\varepsilon_{1}^{e}+\varepsilon_{1}^{\mu}$. The final baryon asymmetry $Y_{B}$ may be approximated as [83]

$$
Y_{B} \simeq-\frac{12}{37 g^{*}}\left[\varepsilon_{1}^{(2)} \eta\left(\frac{417}{589} \tilde{m}_{2}\right)+\varepsilon_{1}^{\tau} \eta\left(\frac{390}{589} \tilde{m}_{\tau}\right)\right],
$$

where the washout masses $\tilde{m}_{2, \tau}$ and $\varepsilon_{1}^{(2)}$ are defined as

$$
\tilde{m}_{2}=M_{1}^{-1}\left(\left|\left(m_{D}\right)_{1 e}\right|^{2}+\left|\left(m_{D}\right)_{1 \mu}\right|^{2}\right), \tilde{m}_{\tau}=M_{1}^{-1}\left|\left(m_{D}\right)_{1 \tau}\right|^{2}, \varepsilon_{1}^{(2)}=\sum_{\alpha=e, \mu} \varepsilon_{1}^{\alpha}=\varepsilon_{1}^{e}+\varepsilon_{1}^{\mu} .
$$


In order to know the nature of the washout processes, it is convenient to define two washout parameters $K_{2, \tau}=\tilde{m}_{2, \tau} / 10^{-3}$ relevant to this mass regime. Further, $\eta\left(\tilde{m}_{2}\right)$ and $\eta\left(\tilde{m}_{\tau}\right)$ are the efficiency factors that account for the inverse decay and the lepton number violating scattering processes while $g^{*}$ is the number of relativistic degrees of freedom in the thermal bath having a value $g^{*} \approx 106.75$ in the SM.

3) Fully flavored leptogenesis: $T \sim M_{1}<10^{9} \mathrm{GeV}$, when in addition to the $\tau$ flavor, the $\mu$ flavor is also in equilibrium - thus all the three flavors are distinguishable. Again for the evaluation of the final baryon asymmetry $Y_{B}$ in this regime, we make use of the approximate analytic formula for $Y_{B}$ presented in ref. [83]. In the $T \sim M_{1}<10^{9} \mathrm{GeV}$ regime, $Y_{B}$ is well approximated by

$$
Y_{B} \simeq-\frac{12}{37 g^{*}}\left[\varepsilon_{1}^{e} \eta\left(\frac{151}{179} \tilde{m}_{e}\right)+\varepsilon_{1}^{\mu} \eta\left(\frac{344}{537} \tilde{m}_{\mu}\right)+\varepsilon_{1}^{\tau} \eta\left(\frac{344}{537} \tilde{m}_{\tau}\right)\right],
$$

where the washout masses $\tilde{m}_{\alpha}$ are defined as

$$
\tilde{m}_{\alpha}=\frac{\left|\left(m_{D}\right)_{1 \alpha}\right|^{2}}{M_{1}}, \alpha=e, \mu, \tau .
$$

We now focus on the calculation of the quantities related to the leptogenesis in our model. The flavor sum over $\alpha$ leads the first term in the r.h.s. of (6.3) to be proportional to $\operatorname{Im}\left(h_{i j}\right)^{2}$ and the second term to vanish. This is since

$$
\sum_{\alpha} \operatorname{Im}\left[h_{j i}\left(m_{D}\right)_{i \alpha}\left(m_{D}^{*}\right)_{j \alpha}\right]=\operatorname{Im}\left[h_{j i} h_{i j}\right]=\operatorname{Im}\left[h_{j i} h_{j i}^{*}\right]=\operatorname{Im}\left|h_{j i}\right|^{2}=0 .
$$

In fact, in our model the matrix $h=m_{D} m_{D}^{\dagger}$ is real as given by

$$
h=\left(\begin{array}{cc}
2\left(a_{1}^{2}+b_{1}^{2}\right) & 2\left(a_{1} a_{2}+b_{1} b_{2} \cos \theta\right) \\
2\left(a_{1} a_{2}+b_{1} b_{2} \cos \theta\right) & 2\left(a_{2}^{2}+b_{2}^{2}\right)
\end{array}\right) .
$$

Therefore the flavor summed CP asymmetry parameter $\varepsilon_{1}=\sum_{\alpha} \varepsilon_{1}^{\alpha}$ vanishes, i.e., unflavored leptogenesis does not occur in this complex (CP) extended $\mu \tau$ antisymmetry scheme. Using (4.4) and (6.3), the flavored CP asymmetries can be calculated to be

$$
\varepsilon_{1}^{e}=0, \varepsilon_{1}^{\mu}=-\frac{g^{\prime}\left(x_{12}\right)}{4 \pi v^{2}}\left[\frac{\left(a_{1} a_{2}+b_{1} b_{2} \cos \theta\right) b_{1} b_{2} \sin \theta}{a_{1}^{2}+b_{1}^{2}}\right]=-\varepsilon_{1}^{\tau},
$$

where $g^{\prime}\left(x_{12}\right)$ is given by

$$
g^{\prime}\left(x_{12}\right)=g\left(x_{12}\right)+\left(1-x_{12}\right)^{-1} .
$$

It is useful to simplify (6.13) for a hierarchical $\mathrm{RH}$ neutrino scheme to

$$
g^{\prime}\left(M_{2}^{2} / M_{1}^{2}\right)=-\frac{3}{2} \frac{M_{1}}{M_{2}}-\frac{M_{1}^{2}}{M_{2}^{2}} .
$$

Now in our minimal seesaw scheme, assuming a specific hierarchy of the $\mathrm{RH}$ neutrino masses, namely $M_{2} / M_{1} \simeq 10^{3}$, the final $Y_{B}$ is calculated from (6.12), (6.6) and (6.8) to be

$$
Y_{B} \simeq \frac{12}{37 g^{*}} \varepsilon_{1}^{\mu}\left[\eta\left(\frac{390}{589} \tilde{m}_{\tau}\right)-\eta\left(\frac{417}{589} \tilde{m}_{2}\right)\right]
$$


for the $\tau$-flavored regime and

$$
Y_{B} \simeq \frac{12}{37 g^{*}} \varepsilon_{1}^{\mu}\left[\eta\left(\frac{344}{537} \tilde{m}_{\tau}\right)-\eta\left(\frac{344}{537} \tilde{m}_{\mu}\right)\right]
$$

for the fully flavored regime.

In our primary analysis, the effect of the heavy neutrino $\left(N_{2}\right)$ on the produced final baryon asymmetry has been neglected with the assumption that the asymmetry produced by the decays of $N_{2}$ get washed out [82]. We now give a brief discussion on how the heavy neutrino $N_{2}$ can affect the final baryon asymmetry $Y_{B}$. As elaborated below, there are two ways in which the effect of $N_{2}$ might arise: indirect and direct. We first discuss the indirect effect. Though the neutrino oscillation data are fitted with the rescaled parameters of (4.6), in order to compute the quantities related to leptogenesis such as $\varepsilon_{1}^{\alpha}$, we need to evaluate the parameters of the Dirac mass matrix elements. Given a set of rescaled parameters, the latter can be generated by varying $M_{1,2}$ in (4.6). It is thus interesting to see whether the final baryon asymmetry is affected by the chosen mass ratios of the $\mathrm{RH}$ neutrinos. We find that the final $Y_{B}$ is not particularly sensitive to $M_{2}$. A relook at (6.14) reminds us that the second term is suppressed compared to the first term, since the former is of the order of $x_{12}^{-1}$. Thus, taking only the first term of (6.14) into consideration, the flavored $\mathrm{CP}$ asymmetry parameters of (6.12) can be simplified in terms of the rescaled parameters of (4.6) as

$$
\varepsilon_{1}^{\mu}=\frac{3 M_{1}}{8 \pi v^{2}}\left[\frac{\left(x_{1} x_{2}+y_{1} y_{2} \cos \theta\right) y_{1} y_{2} \sin \theta}{x_{1}^{2}+y_{1}^{2}}\right]=-\varepsilon_{1}^{\tau} .
$$

Since all rescaled parameters in (6.17) are fixed by the $3 \sigma$ oscillation data, $\varepsilon_{1}^{\mu, \tau}$ are practically insensitive to the value of $M_{2}$. Nevertheless, for a precise numerical computation of the final baryon asymmetry, we need to take into account the effect of the second term in (6.14). The sensitivity of $Y_{B}$ to the magnitude of the second term of (6.14) for different mass hierarchical schemes of the $\mathrm{RH}$ neutrinos will be discussed in detail in the numerical section 7 .

We now turn to discuss the direct effect of $N_{2}$. We have so far focused on the lepton asymmetry produced by the decay of the lightest of the heavy neutrinos. It is shown in ref. [89] that, due to a decoherence effect, the amount of lepton asymmetry, generated by $N_{2}$ decays, gets protected against $N_{1}$-washout. The latter therefore survives down to the electroweak scale and contributes to the final baryon asymmetry. For this procedure to work out, two washout parameters $\Delta_{1}=h_{11} M_{1}^{-1} m^{*-1}$ and $\Delta_{2}=h_{22} M_{2}^{-1} m^{*-1}$ must satisfy the condition

$$
\Delta_{1} \gg 1 \text { and } \Delta_{2} \gg 1
$$

with $m^{*}=1.66 \sqrt{g^{*}} \pi v^{2} / M_{P l} \approx 10^{-3} \mathrm{eV}$. Here $\Delta_{1} \gg 1$ indicates that very fast $N_{1}$ interactions destroy the coherence among the states produced by $N_{2}$; hence a part of the lepton asymmetry produced by $N_{2}$ becomes blind to the $N_{1}$-washout and survives orthogonal to $N_{1}$-states. On the other hand, a mild washout of the lepton asymmetry, produced by $N_{2}$ due to $N_{2}$-related interactions, is represented by the $\Delta_{2} \gg 1$ condition. For such a mild washout scenario, a sizable lepton asymmetry generated by $N_{2}$ survives through 

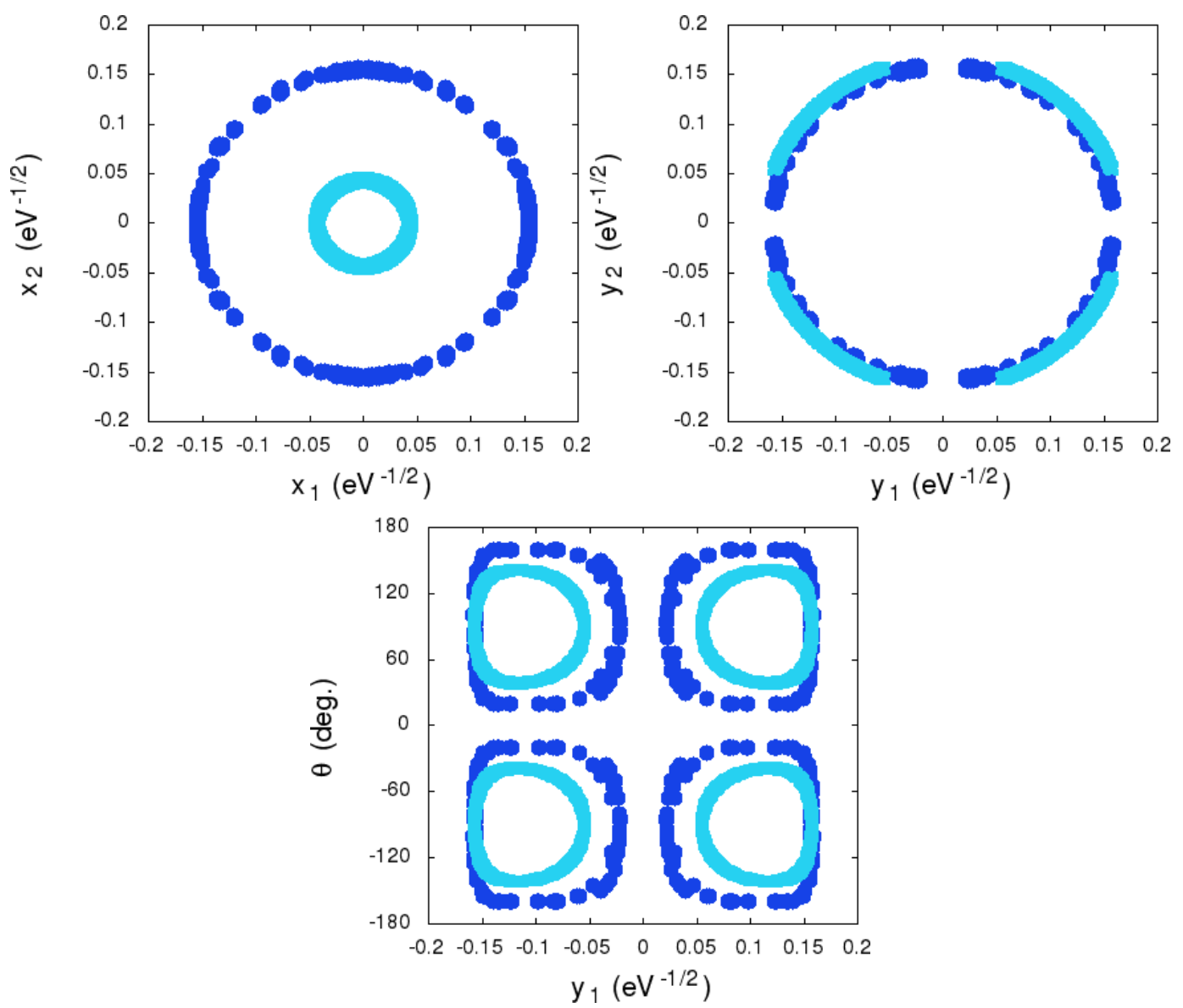

Figure 1. Rescaled parameter space for both the mass orderings. The plots in sky blue (deep blue) color represents the parameter space for normal (inverted) mass ordering.

the $N_{1}$-leptogenesis phase and hence contributes to the final baryon asymmetry. We shall elaborate on the validity of these conditions in our model in the following section.

\section{Numerical analysis: methodology and discussion}

In order to check the viability of our theoretical assumptions and consequent outcomes, we present a numerical analysis in substantial detail. Our method of analysis and organization are as follows. First, we utilize the $3 \sigma$ values of the globally fitted neutrino oscillation data presented in table 2 to constrain the parameter space in terms of the rescaled parameters defined in (4.6). For numerical computation, we make use of the exact analytical formulae for the light neutrino masses and mixing angles presented in ref. [90]. It is seen that, in this complex extended $\mu \tau$ antisymmetry scheme, an appreciable region of the of parameter space could be well fitted within the $3 \sigma$ range of the global oscillation data (see figure 1 ) for each of the mass orderings. We next discuss the predictions of the present model in the context of the $\beta \beta 0 \nu$ experiments for both mass orderings. In order to estimate the value of $Y_{B}$, we make use of these constrained rescaled parameters with a subtlety. For the computation of $Y_{B}$ we need to evaluate the parameters of $m_{D}$ (i.e., $a_{1,2}, b_{1,2}$ ) and 


\begin{tabular}{|c|c|c|c|c|c|}
\hline Parameters & $\sin ^{2} \theta_{12} / 10^{-1}$ & $\sin ^{2} \theta_{23} / 10^{-1}$ & $\sin ^{2} \theta_{13} / 10^{-2}$ & $\begin{array}{c}\Delta m_{21}^{2} / 10^{-5} \\
\left(\mathrm{eV}^{2}\right)\end{array}$ & $\begin{array}{c}\left|\Delta m_{31}^{2}\right| / 10^{-3} \\
\left(\mathrm{eV}^{2}\right)\end{array}$ \\
\hline $3 \sigma$ ranges (NO) & $2.50-3.54$ & $3.81-6.15$ & $1.90-2.40$ & $6.93-7.96$ & $2.411-2.646$ \\
\hline $3 \sigma$ ranges (IO) & $2.50-3.54$ & $3.83-6.36$ & $1.90-2.42$ & $6.93-7.96$ & $2.39-2.624$ \\
\hline Best fit values (NO) & 2.97 & 4.25 & 2.15 & 7.37 & 2.52 \\
\hline Best fit values (IO) & 2.97 & 5.89 & 2.16 & 7.37 & 2.50 \\
\hline
\end{tabular}

Table 2. Input values fed into the analysis [45].

$M_{i}$ separately. Since we have only constrained the rescaled parameters, for a given set of rescaled parameters, there remains a freedom to make various sets of independent choices for the elements of $m_{D}$ along with $M_{i}$. Keeping this in mind, we explore two different numerical ways to discuss leptogenesis and its consequent outcomes. First, we choose a specific hierarchical mass spectrum for the RH neutrinos: $M_{2} / M_{1}=10^{3}$. Then, for a fixed value of $M_{1}$, we use the entire parameter space for the rescaled parameters to generate the elements of $m_{D}$ which are explicitly used to compute the final $Y_{B}$. This leads to a lower bound on $M_{1}$ below which $Y_{B}$ in the observed range cannot be generated. In another approach, instead of taking the entire rescaled parameter space, we focus only on that set of rescaled parameters which corresponds to a positive value of $Y_{B}$ (the sign of $Y_{B}$ depends upon the rescaled parameters) and observables that lie near their best-fit values. Then by varying $M_{1}$, we generate the corresponding parameters of $m_{D}$ using (4.6). Here we consider the same hierarchical scenario for the RH neutrinos as considered in the first approach. Now, for each value of $M_{1}$ and the corresponding parameters of $m_{D}$, we obtain a value for the final baryon asymmetry $Y_{B}$. Since $Y_{B}$ has an observed upper and a lower bound, we end up with an upper and a lower bound for $M_{1}$ also. Finally, we provide a numerical discussion regarding the effects of the heavy neutrino $N_{2}$ on the final $Y_{B}$ as explained analytically in the previous section. We next present the numerical results of our analysis in much more detailed and a systematic way.

As discussed in section 3, there are four sets of $\mathrm{CP}$ violating phases for the four independent $\tilde{d}$ matrices. Thus we get four different plots for each mass of the orderings of the light neutrinos. In figure 2 we present the plots of $\left|M_{e e}\right|$ vs. the sum of the light neutrino masses $\left(\Sigma_{i} m_{i}\right)$ for each mass ordering. Since the lightest neutrino mass is zero in each case, the other two masses $\left(m_{2}\right.$ and $m_{3}$ for normal ordering and $m_{2}$ and $m_{1}$ for inverted ordering) get fixed in a very narrow range by the oscillation constraints on $\Delta m_{21}^{2}$ and $\left|\Delta m_{23}^{2}\right|$. It is evident from figure 2 that $\left|M_{e e}\right|$ in each plot leads to an upper limit which is beyond the reach of the GERDA phase-II. However, predictions of our model could be probed by the combined GERDA + MAJORANA experiments [91]. The sensitivity reach of other promising experiments such as LEGEND-200 (40 meV), LEGEND-1K (17 meV) and nEXO (9 meV) [92] are also shown in figure 2. For each case, the entire parameter space corresponding to an inverted neutrino mass ordering could be ruled out by the nEXO reach.

We now come to the numerical discussion of baryogenesis via flavored leptogenesis. As mentioned in the beginning of this section, we have performed the numerical computation 

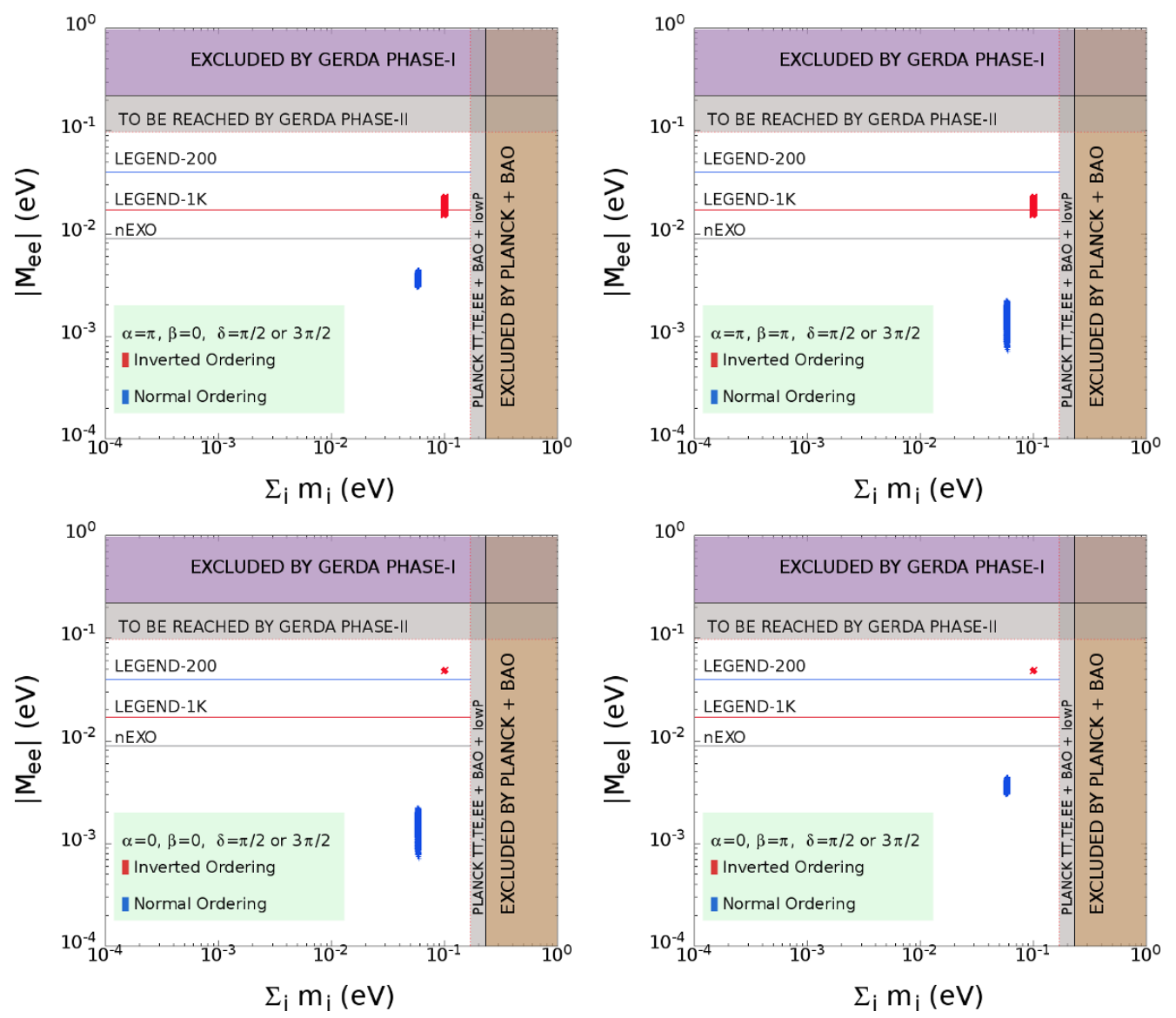

Figure 2. Plots: $\left|M_{e e}\right|$ vs. $\Sigma_{i} m_{i}$ for both the mass orderings.

pertaining to leptogenesis in two different ways. In one way, we have taken a particular value of $M_{1}$ and compute the final $Y_{B}$ for the entire rescaled parameter space constrained by the oscillation data. In the second way, we have used those values of the rescaled parameters for which the low energy neutrino observables predicted from our model lie close to their best fit values dictated by the oscillation data in table 2. To facilitate this purpose, we define a variable $\chi^{2}$ that measures the deviation of the parameters from their best fit values:

$$
\chi^{2}=\sum_{i=1}^{5}\left[\frac{\mathcal{O}_{i}(t h)-\mathcal{O}_{i}(b f)}{\Delta \mathcal{O}_{i}}\right]^{2} .
$$

In (7.1) $\mathcal{O}_{i}$ denotes the $i^{\text {th }}$ neutrino oscillation observable from among $\Delta m_{21}^{2}, \Delta m_{32}^{2}, \theta_{12}, \theta_{23}$ and $\theta_{13}$ and the summation runs over all such observables. The parenthetical th stands for the numerical value of the observable predicted in our model, whereas $b f$ denotes the best fit value (cf. table 2). $\Delta \mathcal{O}_{i}$ in the denominator represents the measured $1 \sigma$ range of $\mathcal{O}_{i}$. Primarily for numerical computation, we choose $M_{2} / M_{1}=10^{3}$. However, as indicated in the previous section, we also present a detailed discussion regarding the sensitivity of $Y_{B}$ to the chosen hierarchy of $M_{i}$. Next, we calculate $\chi^{2}$ as a function of the primed parameters for their entire constrained range. Then, for a fixed value of $M_{1}$, we choose that set of 
rescaled parameters which corresponds to the minimum value of $\chi^{2}$ and a positive value of $Y_{B}$. For that particular $\chi^{2}$ and the corresponding set of rescaled parameters, we are then able to generate a large set of elements of $m_{D}$ by varying $M_{1}$ over a wide range and can calculate $Y_{B}$ for each value of $M_{1}$. An organized discussion is given in what follows.

\section{Computation of $Y_{B}$ for a normal mass ordering of light neutrinos.}

$M_{\mathbf{1}}<\mathbf{1 0}^{\mathbf{9}} \mathbf{G e V}$. In this regime, all three lepton flavors $(e, \mu, \tau)$ are distinguishable. Since $\varepsilon_{1}^{e}=0$, we need to individually evaluate $\varepsilon_{1}^{\mu, \tau}$ only. However, due to the imposed $\mu \tau$ antisymmetry, two washout parameters $\tilde{m}_{\mu}$ and $\tilde{m}_{\tau}$ would be equal. Thus on account of the relation in (6.16), the final baryon asymmetry $Y_{B}$ vanishes.

$10^{9} \mathrm{GeV}<M_{\mathbf{1}}<\mathbf{1 0}^{\mathbf{1 2}} \mathrm{GeV}$. For the evaluation of $Y_{B}$ here, we have to look first at the washout parameters $K_{\tau}$ and $K_{2}=K_{e}+K_{\mu}$. As shown in the first plot in the left panel of figure 3 , the entire allowed range of these parameters prefers to lie in $K_{\tau}, K_{2}>1$ region. Thus the efficiency factor in (6.6) can be written in a strong wash-out scenario [83] as

$$
\eta\left(\tilde{m}_{\alpha}\right)=\left[\left(\frac{0.55 \times 10^{-3}}{\tilde{m}_{\alpha}}\right)^{1.16}\right],
$$

where $\alpha=\tau, 2$. As elaborated in the previous section, the assumed strong hierarchy of $\mathrm{RH}$ neutrinos makes the second r.h.s. term in (6.14) much smaller than the first term. Hence the final CP asymmetry could be simplified to the form as in (6.17) so that the final $Y_{B}$ in (6.15) is practically proportional to the free parameter $M_{1}$. Now for a fixed value of $M_{1}$, we compute $Y_{B}$ for the entire rescaled parameter space. In figure 3 , the variation of $Y_{B}$ with the rescaled parameters is shown for a representative value of $M_{1}=10^{11} \mathrm{GeV}$. Any further lowering of the value of $M_{1}$ would cause these plots (except the first plot in the left panel) to condense along $Y_{B}$ axis due to the addressed proportionality of $Y_{B}$ with $M_{1}$. Thus, below a certain value of $M_{1}$, one would end up with a value for $Y_{B}$ which is below the lower end $8.55 \times 10^{-11}$ of the observed range for the latter. We find this lower bound on $M_{1}$ to be $6.21 \times 10^{10} \mathrm{GeV}$ for which the peak of a $Y_{B}$ vs. $\theta, x_{1,2}, y_{1,2}$ curve in figure 3 just touches the red stripe that represents the experimental observed range of $Y_{B}$.

Next, we concentrate on the other way which is a search for a set of rescaled parameters that corresponds to the low energy neutrino observables close to their best fit values and hence the minimum value of $\chi^{2}$. For this purpose, we take a particular set from the rescaled parameter space, calculate the corresponding $\chi^{2}$ using (7.1) and then compute $Y_{B}$. We have found that $\chi_{\min }^{2}$ should be 0.397 for $Y_{B}$ to be positive. A complete data set of the rescaled parameters and corresponding values of the observables are tabulated in table 3 for $\chi_{\min }^{2}=0.397$.

Given the rescaled data set for the $\chi_{\min }^{2}, M_{1}$ is varied widely to secure $Y_{B}$ in the observed range. For each value of $M_{1}$, a set of values of the parameters in the elements of $m_{D}$ is generated. The final $Y_{B}$ is then calculated for each value of $M_{1}$ and the corresponding parameters of $m_{D}$. A careful surveillance of the plot in figure 4 leads to the conclusion that we can obtain an upper and a lower bound on $M_{1}$ corresponding to the observed constraint on $Y_{B}$. In order to realize this fact more clearly, two straight lines have been drawn 

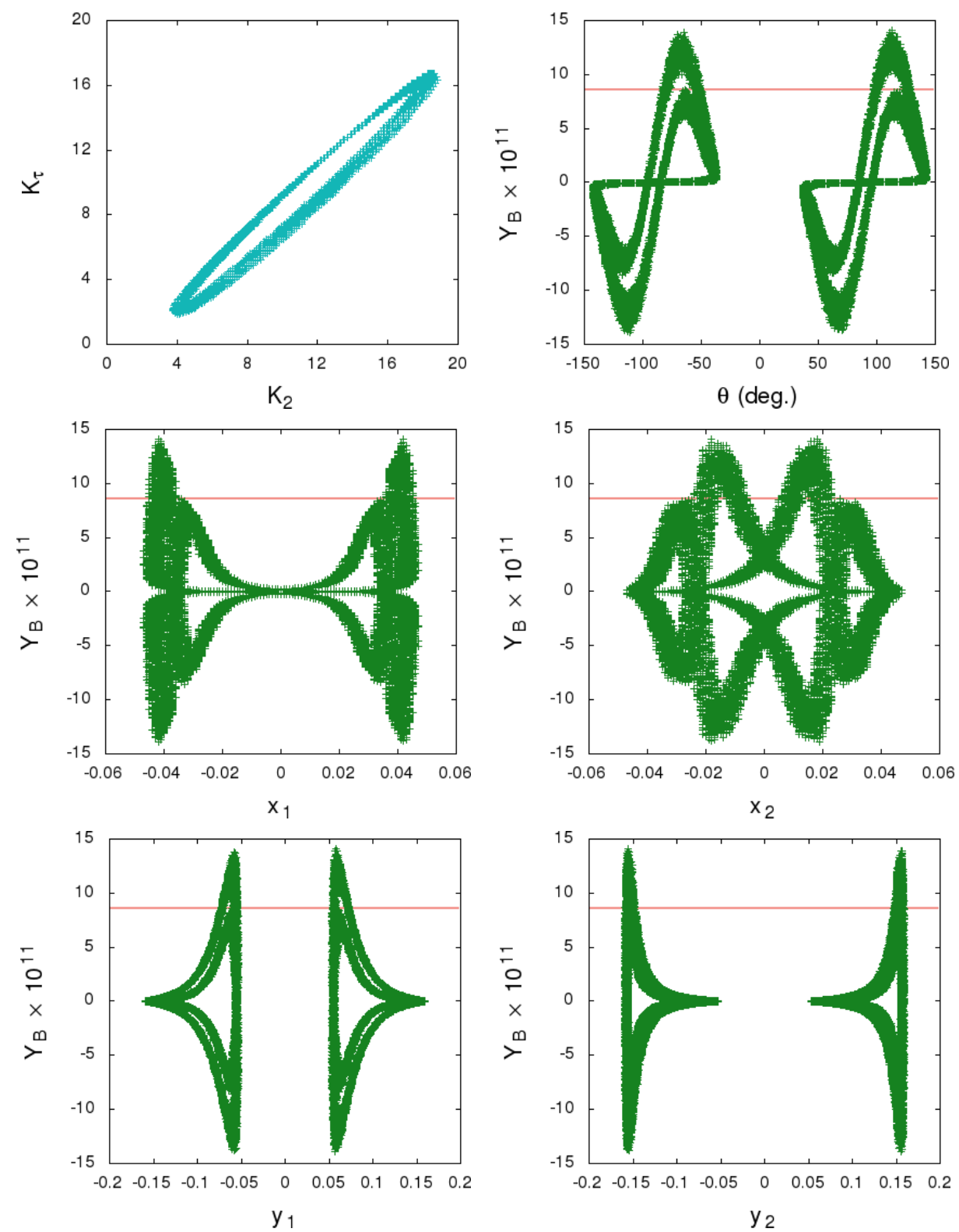

Figure 3. The first figure the left panel shows the ranges for the washout parameters. Rest of the plots represent the variation of $Y_{B}$ with the rescaled parameters for a representative value $M_{1}=10^{11} \mathrm{GeV}$.

\begin{tabular}{|c|c|c|c|c|c|}
\hline$x_{1}$ & $x_{2}$ & $y_{1}$ & $y_{2}$ & $\theta$ & $\chi_{\min }^{2}$ \\
\hline-0.040 & -0.014 & -0.01 & 0.155 & $114^{0}$ & 0.397 \\
\hline \hline \multicolumn{2}{|c|}{ observables } & $\theta_{13}$ & $\theta_{12}$ & $\Delta m_{21}^{2} \times 10^{5}$ & $\left|\Delta m_{31}\right|^{2} \times 10^{3}$ \\
\hline \multicolumn{2}{|c|}{$\chi_{\min }^{2}=0.397$} & $8.42^{0}$ & $33.04^{0}$ & $7.47(\mathrm{eV})^{2}$ & $2.55(\mathrm{eV})^{2}$ \\
\hline
\end{tabular}

Table 3. Parameters and observables corresponding $\chi^{2}=0.397$ for normal mass ordering. 


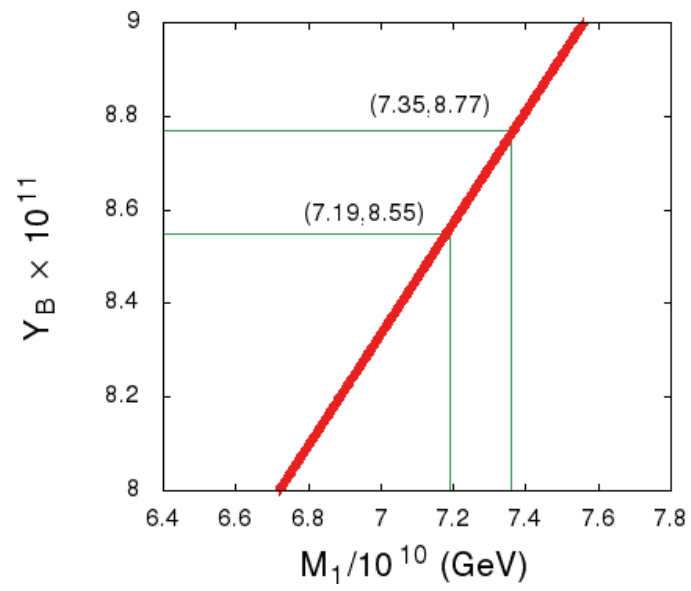

Figure 4. $Y_{B}$ vs. $M_{1}$ curve corresponding to $\chi_{\min }^{2}=0.397$ for a normal mass ordering of the light neutrinos.

parallel to the abscissa in the mentioned plot: one at $Y_{B}=8.55 \times 10^{-11}$ and the other at $Y_{B}=8.77 \times 10^{-11}$. The values of $M_{1}$, where the straight lines connect the $Y_{B}$ vs. $M_{1}$ curve, yield the allowed upper and lower bounds on $M_{1}$, namely $\left(M_{1}\right)_{\text {upper }}=7.35 \times 10^{10} \mathrm{GeV}$ and $\left(M_{1}\right)_{\text {lower }}=7.19 \times 10^{10} \mathrm{GeV}$. Again, the near linearity of the $Y_{B}$ vs. $M_{1}$ curve in figure 4 follows from the previously explained approximate proportionality of $Y_{B}$ with $M_{1}$. One might also ask about the narrow range for $M_{1}$ as observed in figure 4 . Note that in this plot we have presented our result for a particular set of rescaled parameters (with $\chi_{\min }^{2}=0.397$ ). In principle, one could take the entire rescaled parameter space of our model and compute the corresponding results on $Y_{B}$ and $M_{1}$ for each set of the mentioned parameters. In that case the range of $M_{1}$ would not be as narrow as shown in figure 4 .

$M_{1}>\mathbf{1 0}^{\mathbf{1 2}} \mathbf{G e V}$. In this regime $Y_{B}$ is zero since the flavored sum CP asymmetry parameter $\sum_{\alpha} \varepsilon_{1}^{\alpha}$ vanishes. Obviously, $Y_{B}$ might be generated in this regime also if one consider small breaking of CP symmetry in the neutrino sector as discussed in ref. [93].

Computation of $Y_{B}$ for an inverted mass ordering of light neutrinos. In this case also the observed range of $Y_{B}$ cannot be generated for $M_{1}<10^{9} \mathrm{GeV}$ and $M_{1}>10^{12} \mathrm{GeV}$ owing to reasons similar to those explained in the case of a normal ordering. However, we find that in the case of an inverted ordering, $Y_{B}$ cannot be generated in the observed range even if we consider a $\tau$-flavored regime, i.e., $10^{9} \mathrm{GeV}<M_{1}<10^{12} \mathrm{GeV}$. Numerically, for a value $M_{1}=9.9 \times 10^{11} \mathrm{GeV}, Y_{B}$ is computed to be $Y_{B}=8.20 \times 10^{-11}$. Thus from a hierarchical leptogenesis perspective, an inverted mass ordering is disfavored in our model with a complex $(\mathrm{CP})$ extended antisymmetry.

The effect of $N_{2}$ on $Y_{B}$. As mentioned in the previous section, there are two different ways in which the heavy $\mathrm{RH}$ neutrino $N_{2}$ might affect the final value of $Y_{B}$. In the first, which we name as the indirect effect, the final $Y_{B}$ becomes practically insensitive to the mass of $N_{2}$ since the second term is suppressed compared to the first term in (6.14). Now $\varepsilon_{1}^{\mu}$ can be written in a simpler form which is independent of $M_{2}$. c.f, (6.17); hence it does 


\begin{tabular}{|c|c|c|c|}
\hline \multicolumn{4}{|c|}{ Case-I: Normal light neutrino ordering } \\
\hline Hierarchies $\rightarrow$ & $M_{2} / M_{1}=10^{2}$ & $M_{2} / M_{1}=10^{3}$ & $M_{2} / M_{1}=10^{4}$ \\
\hline Upper bound $(\mathrm{GeV})$ & $7.32 \times 10^{10}$ & $7.35 \times 10^{10}$ & $7.38 \times 10^{10}$ \\
\hline Lower bound $(\mathrm{GeV})$ & $7.16 \times 10^{10}$ & $7.19 \times 10^{10}$ & $7.20 \times 10^{10}$ \\
\hline
\end{tabular}

Table 4. Lower and upper bounds on $M_{1}$ for different mass ratios of the RH neutrinos.
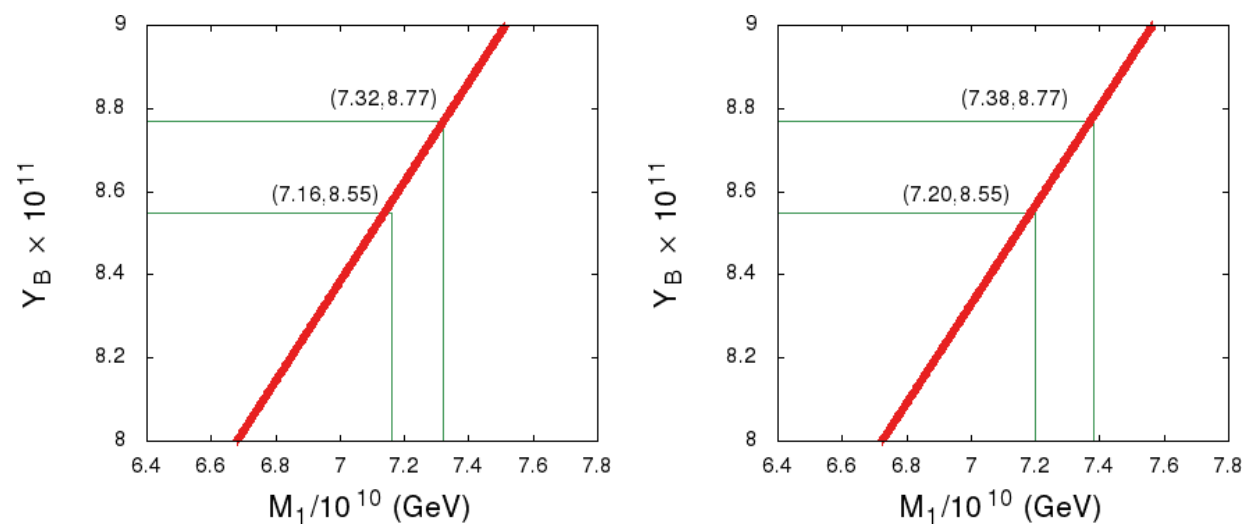

Figure 5. $Y_{B}$ vs. $M_{1}$ plots corresponding to $\chi_{\min }^{2}=0.397$ for the normal mass ordering of the light neutrinos. The plot in the left is for $M_{2} / M_{1}=10^{2}$ and the plot in the right is for $M_{2} / M_{1}=10^{4}$.

not depend the mass ratio $M_{2} / M_{1}$. However, for a precise computation of $Y_{B}$, we need to consider the term neglected in (6.14); that in turn motivates us to perform a quick check of the RH neutrino mass hierarchy sensitivity of the produced value of $Y_{B}$. For this purpose, in addition to the standard hierarchical case, i.e. $M_{2} / M_{1}=10^{3}$, we calculate $Y_{B}$ for two other different mass hierarchical schemes, $M_{2} / M_{1}=10^{2}$ and $M_{2} / M_{2}=10^{4}$. From figure 5 we can infer that though the chosen mass ratios of the $\mathrm{RH}$ neutrinos are altered, changes in the lower and upper bounds on $M_{1}$ are practically insignificant. For the allowed normal light neutrino mass ordering, the variation of $Y_{B}$ with $M_{1}$ for different mass ratios of the $\mathrm{RH}$ neutrinos has been presented in table 4 . It is obvious from the entries of table 4 that a slight difference in the upper and lower bounds on $M_{1}$ in a particular column, as compared to the other column, arises due the dependence of $M_{2}$ on the second term in (6.14). For a fixed value of $M_{1}$, the contribution from the second term in (6.14) is larger for $M_{2} / M_{1}=10^{2}$ and smaller for $M_{2} / M_{1}=10^{4}$, as compared to the standard $M_{2} / M_{1}=10^{3}$ case. Hence for $M_{2} / M_{1}=10^{2}$, the slope of the $Y_{B}$ vs. $M_{1}$ curve is larger than for $M_{2} / M_{1}=10^{3}$. Consequently, for the allowed range of $Y_{B}$, both the upper and the lower bounds get slightly left shifted on the $M_{1}$-axis (compared to the standard $M_{2} / M_{1}=10^{3}$ case). Proceeding in the same way, we obtain somewhat right shifted bounds for $M_{2} / M_{1}=10^{4}$ case.

In contrast, in the direct effect, any asymmetry produced by $N_{2}$ survives provided the conditions $\Delta_{1} \gg 1$ and $\Delta_{2} \gg 1$, cf. (6.18), are satisfied. From figure 6 we observe that the allowed parametric region prefers large values of $\Delta_{2}$ in excess of 10 except at the bottom (green band). Thus the condition $\Delta_{2} \gg 1$ is violated in most of the region. Moreover the $\chi_{\min }^{2}=0.397$, for which we calculate final $Y_{B}$ strongly violates $\Delta_{2} \ngtr 1$ condition. A tiny 


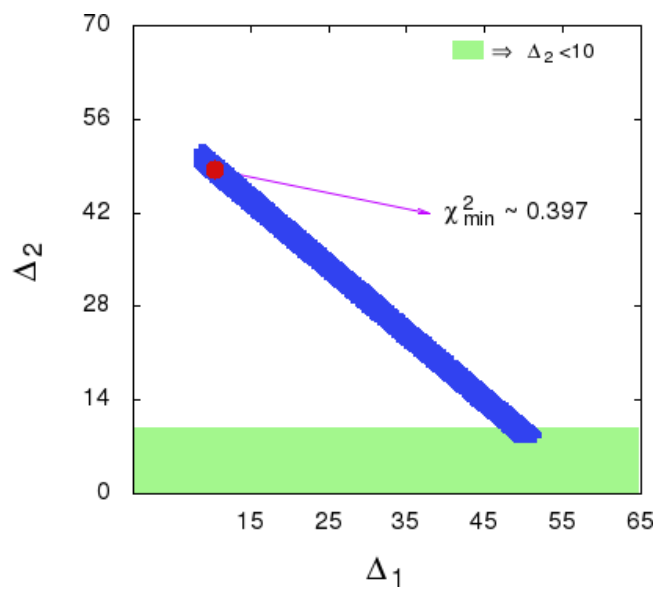

Figure 6. Washout parameters for $N_{2}$ leptogenesis.

amount of parameter space with $\Delta_{2}<10$ corresponds to values of $\chi^{2}$ above 0.9 which is much higher than $\chi_{\min }^{2}$ for which we compute $Y_{B}$ in the observed range. Therefore, in our final result, any direct effect of $N_{2}$ is not significant. Note that there is nothing special about $\chi^{2}=0.9$. The issue we are trying to address here, is that there are indeed some data points in the model parameter space for which the conditions for $N_{2}$ leptogenesis could be satisfied. However, the minimum value of $\chi^{2}$ for those data sets is 0.9 . This means that the corresponding observables are away from their best-fit values (though well within $1 \sigma$ ) and thus the obtained bounds on $M_{1}$ (e.g. figure 4 ) will not be affected by $N_{2}$ leptogenesis. However, if one goes beyond $\chi^{2} \approx 0.9$, the asymmetry produced by $N_{2}$ could play a crucial role.

We would like to conclude this section by comparing our results on leptogenesis with those obtained earlier in previous literature in case of a $\mu \tau$ flavored CP symmetry. Existing references such as $[42,72,73,93]$ also discuss leptogenesis within the framework of residual $\mathrm{CP}$ symmetry (in particular $\mathrm{CP}^{\mu \tau}$ ) and point out the nonoccurrence of unflavored leptogenesis and only the viability of the $\tau$-flavored scenario similar to our proposal of a exact $\mu \tau$ antisymmetry in the neutrino sector. However, the final numerical analysis is different from our case. In particular, all the mentioned references mainly focus on the three neutrino case where one cannot fix the Yukawa couplings only with the oscillation data. Thus any final result on leptogenesis requires other assumptions to constrain all the Yukawas. We focus on the two RH neutrino case, namely the minimal seesaw mechanism, where the entire Yukawa parameter (rescaled by RH neutrino masses) space could be constrained by the neutrino oscillation data. Hence all the results obtained, in particular for the $\mathrm{RH}$ neutrino masses, are exactly dictated by the oscillation data. For a hierarchical RH mass spectrum, refs. $[72,73]$ shows a variation of $Y_{B}$ with a single model parameter for a fixed value of $M_{1}$ and best-fit values of the oscillation parameters. However, here we focus on the bounds on $M_{1}$ for the entire parameter space as well as the for the parameter set that corresponds to the observables which lie near to their best-fit values. For the first case, we obtain a lower bound on $M_{1}$ while in the other, we obtain an upper as well as a lower bound on $M_{1}$. In addition, we have done a thorough study of the RH neutrino hierarchy 
sensitivity of the final $Y_{B}$ and showed the possible changes in the bounds on the lightest $\mathrm{RH}$ neutrino $M_{1}$ for three different $\mathrm{RH}$ neutrino hierarchical mass spectra. We have also showed that, for this minimal seesaw with a complex $\mu \tau$ antisymmetry, the inverted mass ordering is not a viable option as far as hierarchical leptogenesis is concerned. We are not within the framework of a Grand Unified Theory (GUT) such as SO(10), where the lepton asymmetry generated by the next to light $\mathrm{RH}$ neutrino $\left(N_{2}\right)$, is a natural requirement to produce correct value of $Y_{B}[94,95]$. Nevertheless, we opt for fast $N_{1}$ interactions which are responsible for the survival of the lepton asymmetry generated by $N_{2}$ [89]. For this CP symmetric framework we have showed for the first time that there could be a parameter space left for which $N_{2}$ leptogenesis might affect the final value of $Y_{B}$ (though a rigorous study of the $N_{2}$ leptogenesis is beyond the scope of this paper). Ref. [42] concluded that for the mass regime $M_{1}<10^{9} \mathrm{GeV}$, a resonant leptogenesis is only possible if one considers breaking in $C P^{\mu \tau}$, since in this regime the muon and tauon washout parameters are of equal strength. In our proposal of $C P^{\mu \tau A}$ also, this conclusion is true. However, as we show in the appendix, unlike the hierarchical $\mathrm{RH}$ neutrino mass spectrum, $\mathrm{RH}$ neutrinos with a mild hierarchy could also result in a successful leptogenesis for $M_{1} \approx 10^{9} \mathrm{GeV}$. We showed that an inverted mass ordering could then be a viable option for a successful leptogenesis. We also comment on the strength of the mild hierarchy by solving numerically the formulae for $Y_{B}$ within the framework of flavor diagonal RH neutrinos. In our analysis, the sign of $Y_{B}$ depends upon the Yukawa parameters. In this context we refer to [93] which shows how, within the framework of a CP symmetry, the sign of $Y_{B}$ depends upon the observables.

\section{Concluding comments and discussion}

In this paper the complex (CP) extension of $\mu \tau$ antisymmetry has been shown to yield a $M_{\nu}^{\mathrm{CP}^{\mu \tau A}}$ which is simply related to $M_{\nu}^{\mathrm{CP}}$ - the Majorana mass matrix from the complex (CP) extension of $\mu \tau$ symmetry with both having identical phenomenological consequences. These phenomenological consequences of $C P^{\mu \tau A}$ have been worked out within a minimal seesaw scheme with two strongly or mildly hierarchical RH neutrinos $N_{1}$ and $N_{2}$. We have further investigated baryogenesis via leptogenesis in this scenario and derived upper and lower bounds on the mass of $N_{1}$.

To summarize, we have proposed a new idea, namely a complex extended $\mu \tau$ antisymmetry, pertaining to the neutrino sector and have worked out its consequences. Unlike the real $\mu \tau$ antisymmetry, we envisage there is no need for any breaking of it in the neutrino sector. Atmospheric neutrino mixing is predicted to be maximal $\left(\theta_{23}=\pi / 4\right)$ in this scheme while the solar and reactor mixing angles $\left(\theta_{12}\right.$ and $\theta_{13}$ respectively) can be fit to their observed values. Neutrino masses get generated via the minimal seesaw mechanism with two heavy right-chiral neutrinos. The lightest neutrino is predicted to be massless while the two other neutrino masses can be fit to the observed range of values of $\left|\Delta m_{32}^{2}\right|$ and $\Delta m_{12}^{2}$ both for a normal and an inverted mass ordering. Concrete predictions are made for neutrinoless double beta decay: the ongoing experiments are not expected to observe it though the planned nEXO experiment may have a chance to do so. Finally, we have made a 
detailed quantitative examination of baryogenesis via leptogenesis in our scheme including the indirect and direct effects of the heavier $\mathrm{RH}$ neutrino $N_{2} . \tau$-flavored leptogenesis with a normal mass ordering turns out to be the only viable possibility that can generate $Y_{B}$ in the observed range in a hierarchical leptogenesis scenario.

\section{Acknowledgments}

The work of RS and AG is supported by the Department of Atomic Energy (DAE), Government of India. The work of PR has been supported by the Indian National Science Academy.

\section{A Discussion of the case with mildly hierarchical RH neutrinos}

In the text we have dealt with a strongly hierarchical $\mathrm{RH}$ neutrino mass spectrum and found only the $\tau$-flavored regime to be viable in producing the correct $Y_{B}$ for a normal light neutrino mass ordering. Since in our chosen basis [72, 73], RH neutrinos are nondegenerate, it would also be interesting to study leptogenesis with a mildly hierarchical including a quasidegenerate $N_{R}$ mass spectrum. We will see later in this discussion that RH neutrinos which are not strongly hierarchical might obliterate all the new bounds on $M_{1}$ that we obtained earlier.

In general, a quasidegenerate RH neutrino mass spectrum is considered for studying leptogenesis in a low energy seesaw scenario (resonant leptogenesis [81]); here the RH neutrinos could have masses $\mathcal{O}(\mathrm{TeV})$. However, in our analysis, we cannot lower the RH neutrino masses below $10^{9} \mathrm{GeV}$, since that would correspond to the fully flavored regime where the two washout parameters $\tilde{m}_{\mu}$ and $\tilde{m}_{\tau}$ are the same due to the imposed $\mu \tau$ antisymmetry, thereby implying a vanishing $Y_{B}$ cf. (6.16). However, depending on the chosen mild mass splitting of the RH neutrinos, we can lower the lightest RH neutrino mass down to $10^{9} \mathrm{GeV}$ below which the muon charged lepton flavor equilibriates. In scenarios where the RH neutrinos are not strongly hierarchical, instead of (6.3), it is useful to use the general formula for the $\mathrm{CP}$ asymmetry parameter [81] $\varepsilon_{i}^{\alpha}$ as

$$
\begin{aligned}
\varepsilon_{i}^{\alpha}= & \frac{1}{4 \pi v^{2} h_{i i}} \sum_{j \neq i} \operatorname{Im}\left\{h_{i j}\left(m_{D}\right)_{i \alpha}\left(m_{D}^{*}\right)_{j \alpha}\right\}\left[f\left(x_{i j}\right)+\frac{\sqrt{x_{i j}}\left(1-x_{i j}\right)}{\left(1-x_{i j}\right)^{2}+h_{j j}^{2}\left(16 \pi^{2} v^{4}\right)^{-1}}\right] \\
& +\frac{1}{4 \pi v^{2} h_{i i}} \sum_{j \neq i} \frac{\left(1-x_{i j}\right) \operatorname{Im}\left\{h_{j i}\left(m_{D}\right)_{i \alpha}\left(m_{D}^{*}\right)_{j \alpha}\right\}}{\left(1-x_{i j}\right)^{2}+h_{j j}^{2}\left(16 \pi^{2} v^{4}\right)^{-1}} .
\end{aligned}
$$

Note that, unlike (6.3) the above equation is valid for degenerate $\mathrm{RH}$ neutrinos also.

Taking into account the contribution from both the $\mathrm{RH}$ neutrinos, we have performed a numerical study to find the final $Y_{B}$ for the lowest allowed value of $M_{1}\left(=10^{9} \mathrm{GeV}\right)$. It turns out that for a normal light neutrino mass ordering, $M_{2}$ could at most be $\approx 17.5 M_{1}$ to produce the observed lower bound $8.55 \times 10^{-11}$ of $Y_{B}$ cf. (6.1). One can see that the obtained mass spectrum is fairly hierarchical though the hierarchy is not very strong. Of course any number smaller than 17.5 would result in an enhancement of the produced CP 
asymmetry. Thus the observed range of $Y_{B}$ could be generated with a quasidegenerate $\mathrm{RH}$ mass spectrum too. Interestingly, an inverted light neutrino mass ordering which is disfavoured for a strongly hierarchical $\mathrm{RH}$ neutrino mass spectrum is now a perfectly viable scenario since we relax the strong hierarchy assumption. Again, as in the previous case, i.e., for $M_{1}=10^{9} \mathrm{GeV}$, it is numerically found that one needs $M_{2} \leqslant 1.8 M_{1}$ in order to produce the observed lower bound on $Y_{B}$. Note that, unlike in the case of a normal light neutrino mass ordering, the $\mathrm{RH}$ neutrino mass spectrum here favours a mild hierarchical scenario as we lower the value of $M_{1}$. We could also point out that here we have considered the flavor diagonal RH neutrinos to calculate the asymmetry. Nevertheless, for a resonant leptogenesis scenario, a full flavor-covariant treatment might play an important role [84-86].

As a concluding remark, we may mention once again that, owing to the imposed symmetry, a fully flavored leptogenesis is not possible for $M_{1}<10^{9} \mathrm{GeV}$ even if we consider strongly degenerate RH neutrinos. Nevertheless, a small breaking of the symmetry [93], or somewhat a more moderate version of the symmetry such as the scaling ansatz [46-48] will cause a deviation from $\tilde{m}_{\mu}=\tilde{m}_{\tau}$ cf. (6.16) and will imply a nonvanishing $Y_{B}$. In such cases leptogenesis with heavily degenerate $\mathrm{RH}$ neutrinos (resonant leptogenesis) could be an interesting topic to study.

Open Access. This article is distributed under the terms of the Creative Commons Attribution License (CC-BY 4.0), which permits any use, distribution and reproduction in any medium, provided the original author(s) and source are credited.

\section{References}

[1] S.F. King, Models of Neutrino Mass, Mixing and CP-violation, J. Phys. G 42 (2015) 123001 [arXiv: 1510.02091] [INSPIRE].

[2] Planck collaboration, P.A.R. Ade et al., Planck 2015 results. XIII. Cosmological parameters, Astron. Astrophys. 594 (2016) A13 [arXiv:1502.01589] [INSPIRE].

[3] S. Vagnozzi et al., Unveiling $\nu$ secrets with cosmological data: neutrino masses and mass hierarchy, Phys. Rev. D 96 (2017) 123503 [arXiv:1701.08172] [INSPIRE].

[4] T2K collaboration, K. Abe et al., Combined Analysis of Neutrino and Antineutrino Oscillations at T2K, Phys. Rev. Lett. 118 (2017) 151801 [arXiv:1701.00432] [INSPIRE].

[5] P. Minkowski, $\mu \rightarrow$ er at a Rate of One Out of $10^{9}$ Muon Decays?, Phys. Lett. B 67 (1977) 421 [INSPIRE].

[6] M. Gell-Mann, P. Ramond and R. Slansky, Complex Spinors and Unified Theories, Conf. Proc. C 790927 (1979) 315 [arXiv: 1306.4669] [INSPIRE].

[7] T. Yanagida, Horizontal Symmetry and Masses of Neutrinos, Prog. Theor. Phys. 64 (1980) 1103 [INSPIRE].

[8] R.N. Mohapatra, Mechanism for Understanding Small Neutrino Mass in Superstring Theories, Phys. Rev. Lett. 56 (1986) 561 [InSPIRE].

[9] S.F. King, Large mixing angle $M S W$ and atmospheric neutrinos from single right-handed neutrino dominance and U(1) family symmetry, Nucl. Phys. B 576 (2000) 85 [hep-ph/9912492] [INSPIRE]. 
[10] S.F. King, Constructing the large mixing angle MNS matrix in seesaw models with right-handed neutrino dominance, JHEP 09 (2002) 011 [hep-ph/0204360] [INSPIRE].

[11] P.H. Frampton, S.L. Glashow and T. Yanagida, Cosmological sign of neutrino CP-violation, Phys. Lett. B 548 (2002) 119 [hep-ph/0208157] [INSPIRE].

[12] M. Raidal and A. Strumia, Predictions of the most minimal seesaw model, Phys. Lett. B 553 (2003) 72 [hep-ph/0210021] [INSPIRE].

[13] V. Barger, D.A. Dicus, H.-J. He and T.-j. Li, Structure of cosmological CP-violation via neutrino seesaw, Phys. Lett. B 583 (2004) 173 [hep-ph/0310278] [INSPIRE].

[14] S. Antusch, P. Di Bari, D.A. Jones and S.F. King, Leptogenesis in the Two Right-Handed Neutrino Model Revisited, Phys. Rev. D 86 (2012) 023516 [arXiv:1107.6002] [INSPIRE].

[15] J. Zhang and S. Zhou, A Further Study of the Frampton-Glashow-Yanagida Model for Neutrino Masses, Flavor Mixing and Baryon Number Asymmetry, JHEP 09 (2015) 065 [arXiv: 1505.04858] [INSPIRE].

[16] F. Björkeroth, F.J. de Anda, I. de Medeiros Varzielas and S.F. King, Leptogenesis in minimal predictive seesaw models, JHEP 10 (2015) 104 [arXiv:1505.05504] [INSPIRE].

[17] G.-J. Ding, S.F. King and C.-C. Li, Golden Littlest Seesaw, Nucl. Phys. B 925 (2017) 470 [arXiv: 1705.05307] [INSPIRE].

[18] T. Kitabayashi and M. Yasuè, Maximal CP-violation in Minimal Seesaw Model, Phys. Rev. D 94 (2016) 075020 [arXiv: 1605.04402] [INSPIRE].

[19] Z.-C. Liu, C.-X. Yue and Z.-h. Zhao, Neutrino $\mu-\tau$ reflection symmetry and its breaking in the minimal seesaw, JHEP 10 (2017) 102 [arXiv:1707.05535] [INSPIRE].

[20] Y. Shimizu, K. Takagi and M. Tanimoto, Towards the minimal seesaw model via CP-violation of neutrinos, JHEP 11 (2017) 201 [arXiv:1709.02136] [INSPIRE].

[21] Y. Shimizu, K. Takagi and M. Tanimoto, Neutrino CP-violation and sign of baryon asymmetry in the minimal seesaw model, Phys. Lett. B 778 (2018) 6 [arXiv:1711.03863] [INSPIRE].

[22] R.N. Mohapatra and S. Nussinov, Bimaximal neutrino mixing and neutrino mass matrix, Phys. Rev. D 60 (1999) 013002 [hep-ph/9809415] [INSPIRE].

[23] T. Fukuyama and H. Nishiura, Mass matrix of Majorana neutrinos, hep-ph/9702253 [INSPIRE].

[24] C.S. Lam, A 2-3 symmetry in neutrino oscillations, Phys. Lett. B 507 (2001) 214 [hep-ph/0104116] [INSPIRE].

[25] E. Ma and M. Raidal, Neutrino mass, muon anomalous magnetic moment and lepton flavor nonconservation, Phys. Rev. Lett. 87 (2001) 011802 [Erratum ibid. 87 (2001) 159901] [hep-ph/0102255] [INSPIRE].

[26] K.R.S. Balaji, W. Grimus and T. Schwetz, The Solar LMA neutrino oscillation solution in the Zee model, Phys. Lett. B 508 (2001) 301 [hep-ph/0104035] [INSPIRE].

[27] A. Ghosal, An $\mathrm{SU}(2)_{L} \times \mathrm{U}(1)_{Y}$ model with reflection symmetry in view of recent neutrino experimental result, hep-ph/0304090 [INSPIRE].

[28] A. Ghosal, A neutrino mass model with reflection symmetry, Mod. Phys. Lett. A 19 (2004) 2579 [INSPIRE]. 
[29] S.-F. Ge, H.-J. He and F.-R. Yin, Common Origin of Soft mu-tau and CP Breaking in Neutrino Seesaw and the Origin of Matter, JCAP 05 (2010) 017 [arXiv:1001.0940] [INSPIRE].

[30] Z.-z. Xing and Z.-h. Zhao, A review of $\mu-\tau$ flavor symmetry in neutrino physics, Rept. Prog. Phys. 79 (2016) 076201 [arXiv: 1512.04207] [INSPIRE].

[31] S.-F. Ge, H.-J. He and F.-R. Yin, Common Origin of Soft mu-tau and CP Breaking in Neutrino Seesaw and the Origin of Matter, JCAP 05 (2010) 017 [arXiv:1001.0940] [INSPIRE].

[32] P.F. Harrison and W.G. Scott, $\mu-\tau$ reflection symmetry in lepton mixing and neutrino oscillations, Phys. Lett. B $5 \mathbf{4 7}$ (2002) 219 [hep-ph/0210197] [INSPIRE].

[33] W. Grimus and L. Lavoura, A nonstandard CP transformation leading to maximal atmospheric neutrino mixing, Phys. Lett. B 579 (2004) 113 [hep-ph/0305309] [INSPIRE].

[34] W. Grimus and L. Lavoura, $\mu-\tau$ Interchange symmetry and lepton mixing, Fortsch. Phys. 61 (2013) 535 [arXiv:1207.1678] [INSPIRE].

[35] W. Grimus, S. Kaneko, L. Lavoura, H. Sawanaka and M. Tanimoto, $\mu-\tau$ antisymmetry and neutrino mass matrices, JHEP 01 (2006) 110 [hep-ph/0510326] [INSPIRE].

[36] Particle Data Group collaboration, K.A. Olive et al., Review of Particle Physics, Chin. Phys. C 38 (2014) 090001 [INSPIRE].

[37] G. Altarelli and F. Feruglio, Discrete Flavor Symmetries and Models of Neutrino Mixing, Rev. Mod. Phys. 82 (2010) 2701 [arXiv:1002.0211] [InSPIRE].

[38] H. Ishimori, T. Kobayashi, H. Ohki, Y. Shimizu, H. Okada and M. Tanimoto, Non-Abelian Discrete Symmetries in Particle Physics, Prog. Theor. Phys. Suppl. 183 (2010) 1 [arXiv: 1003.3552] [INSPIRE].

[39] S.F. King, Unified Models of Neutrinos, Flavour and CP-violation, Prog. Part. Nucl. Phys. 94 (2017) 217 [arXiv: 1701.04413] [INSPIRE].

[40] S.T. Petcov, Discrete Flavour Symmetries, Neutrino Mixing and Leptonic CP-violation, arXiv: 1711.10806 [INSPIRE].

[41] Daya Bay collaboration, F.P. An et al., New Measurement of Antineutrino Oscillation with the Full Detector Configuration at Daya Bay, Phys. Rev. Lett. 115 (2015) 111802 [arXiv: 1505.03456] [INSPIRE].

[42] R.N. Mohapatra and C.C. Nishi, Implications of $\mu$ - $\tau$ flavored CP symmetry of leptons, JHEP 08 (2015) 092 [arXiv: 1506.06788] [INSPIRE].

[43] A.S. Joshipura, Neutrino masses and mixing from flavour antisymmetry, JHEP 11 (2015) 186 [arXiv: 1506.00455] [INSPIRE].

[44] A.S. Joshipura and N. Nath, Neutrino masses and mixing in $A_{5}$ with flavor antisymmetry, Phys. Rev. D 94 (2016) 036008 [arXiv: 1606.01697] [INSPIRE].

[45] F. Capozzi, E. Di Valentino, E. Lisi, A. Marrone, A. Melchiorri and A. Palazzo, Global constraints on absolute neutrino masses and their ordering, Phys. Rev. D 95 (2017) 096014 [arXiv: 1703.04471] [INSPIRE].

[46] R. Samanta, P. Roy and A. Ghosal, Extended scaling and residual flavor symmetry in the neutrino Majorana mass matrix, Eur. Phys. J. C 76 (2016) 662 [arXiv:1604.06731] [INSPIRE]. 
[47] R. Samanta, P. Roy and A. Ghosal, Complex Scaling in Neutrino Mass Matrix, Acta Phys. Polon. Supp. 9 (2016) 807 [arXiv: 1604.01206] [InSPIRE].

[48] R. Sinha, R. Samanta and A. Ghosal, Generalized $\mathbb{Z}_{2} \times \mathbb{Z}_{2}$ in scaling neutrino Majorana mass matrix and baryogenesis via flavored leptogenesis, JHEP 12 (2017) 030 [arXiv: 1706. 00946] [INSPIRE].

[49] G. Ecker, W. Grimus and H. Neufeld, A Standard Form for Generalized CP Transformations, J. Phys. A 20 (1987) L807 [inSPIRE].

[50] H. Neufeld, W. Grimus and G. Ecker, Generalized CP Invariance, Neutral Flavor Conservation and the Structure of the Mixing Matrix, Int. J. Mod. Phys. A 3 (1988) 603 [INSPIRE].

[51] W. Grimus and M.N. Rebelo, Automorphisms in gauge theories and the definition of CP and P, Phys. Rept. 281 (1997) 239 [hep-ph/9506272] [INSPIRE].

[52] R.N. Mohapatra and C.C. Nishi, $S_{4}$ Flavored CP Symmetry for Neutrinos, Phys. Rev. D 86 (2012) 073007 [arXiv: 1208.2875] [INSPIRE].

[53] S. Gupta, A.S. Joshipura and K.M. Patel, Minimal extension of tri-bimaximal mixing and generalized $Z_{2} \times Z_{2}$ symmetries, Phys. Rev. D 85 (2012) 031903 [arXiv:1112.6113] [INSPIRE].

[54] F. Feruglio, C. Hagedorn and R. Ziegler, Lepton Mixing Parameters from Discrete and CP Symmetries, JHEP 07 (2013) 027 [arXiv: 1211.5560] [INSPIRE].

[55] M. Holthausen, M. Lindner and M.A. Schmidt, CP and Discrete Flavour Symmetries, JHEP 04 (2013) 122 [arXiv:1211.6953] [INSPIRE].

[56] M.-C. Chen, M. Fallbacher, K.T. Mahanthappa, M. Ratz and A. Trautner, CP Violation from Finite Groups, Nucl. Phys. B 883 (2014) 267 [arXiv:1402.0507] [InSPIRE].

[57] G.-J. Ding, S.F. King, C. Luhn and A.J. Stuart, Spontaneous CP-violation from vacuum alignment in $S_{4}$ models of leptons, JHEP 05 (2013) 084 [arXiv: 1303.6180] [INSPIRE].

[58] G.-J. Ding, S.F. King and A.J. Stuart, Generalised CP and $A_{4}$ Family Symmetry, JHEP 12 (2013) 006 [arXiv:1307.4212] [INSPIRE].

[59] F. Feruglio, C. Hagedorn and R. Ziegler, A realistic pattern of lepton mixing and masses from $S_{4}$ and $C P$, Eur. Phys. J. C 74 (2014) 2753 [arXiv:1303.7178] [INSPIRE].

[60] P. Chen, C.-Y. Yao and G.-J. Ding, Neutrino Mixing from CP Symmetry, Phys. Rev. D 92 (2015) 073002 [arXiv: 1507.03419] [INSPIRE].

[61] C.C. Nishi, New and trivial CP symmetry for extended $A_{4}$ flavor, Phys. Rev. D 93 (2016) 093009 [arXiv: 1601.00977] [INSPIRE].

[62] C.C. Nishi and B.L. Sánchez-Vega, Mu-tau reflection symmetry with a texture-zero, JHEP 01 (2017) 068 [arXiv:1611.08282] [INSPIRE].

[63] W. Rodejohann and X.-J. Xu, Trimaximal $\mu-\tau$ reflection symmetry, Phys. Rev. D 96 (2017) 055039 [arXiv: 1705. 02027] [INSPIRE].

[64] J.T. Penedo, S.T. Petcov and A.V. Titov, Neutrino mixing and leptonic CP-violation from $S_{4}$ flavour and generalised CP symmetries, JHEP 12 (2017) 022 [arXiv:1705.00309] [INSPIRE].

[65] S.F. King, Unified Models of Neutrinos, Flavour and CP-violation, Prog. Part. Nucl. Phys. 94 (2017) 217 [arXiv:1701.04413] [INSPIRE]. 
[66] J. Bernabeu, G.C. Branco and M. Gronau, CP Restrictions on Quark Mass Matrices, Phys. Lett. B 169 (1986) 243 [INSPIRE].

[67] G.C. Branco, L. Lavoura and M.N. Rebelo, Majorana Neutrinos and CP Violation in the Leptonic Sector, Phys. Lett. B 180 (1986) 264 [INSPIRE].

[68] G.C. Branco, L. Lavoura and J.P. Silva, CP Violation, Clarendon Press, Oxford (1999).

[69] J. Iizuka, Y. Kaneko, T. Kitabayashi, N. Koizumi and M. Yasue, CP violation in modified bipair neutrino mixing and leptogenesis, Phys. Lett. B 732 (2014) 191 [arXiv:1404.0735] [INSPIRE].

[70] R. Samanta and A. Ghosal, Probing maximal zero textures with broken cyclic symmetry in inverse seesaw, Nucl. Phys. B 911 (2016) 846 [arXiv:1507.02582] [INSPIRE].

[71] R. Samanta, M. Chakraborty and A. Ghosal, Evaluation of the Majorana Phases of a General Majorana Neutrino Mass Matrix: Testability of hierarchical Flavour Models, Nucl. Phys. B 904 (2016) 86 [arXiv:1502.06508] [INSPIRE].

[72] P. Chen, G.-J. Ding and S.F. King, Leptogenesis and residual CP symmetry, JHEP 03 (2016) 206 [arXiv: 1602.03873] [InSPIRE].

[73] R. Samanta, M. Chakraborty, P. Roy and A. Ghosal, Baryon asymmetry via leptogenesis in a neutrino mass model with complex scaling, JCAP 03 (2017) 025 [arXiv:1610.10081] [INSPIRE].

[74] KamLAND-Zen collaboration, K. Asakura et al., Search for double-beta decay of ${ }^{136}$ Xe to excited states of ${ }^{136} \mathrm{Ba}$ with the KamLAND-Zen experiment, Nucl. Phys. A 946 (2016) 171 [arXiv: 1509.03724] [INSPIRE].

[75] EXO-200 collaboration, M. Auger et al., Search for Neutrinoless Double-Beta Decay in ${ }^{136}$ Xe with EXO-200, Phys. Rev. Lett. 109 (2012) 032505 [arXiv: 1205.5608] [INSPIRE].

[76] GERDA collaboration, B. Majorovits, The search for $0 \nu \beta \beta$ decay with the GERDA experiment: Status and prospects, AIP Conf. Proc. 1672 (2015) 110003 [arXiv:1506. 00415] [INSPIRE].

[77] M. Fukugita and T. Yanagida, Baryogenesis Without Grand Unification, Phys. Lett. B 174 (1986) 45 [INSPIRE].

[78] A. Riotto and M. Trodden, Recent progress in baryogenesis, Ann. Rev. Nucl. Part. Sci. 49 (1999) 35 [hep-ph/9901362] [INSPIRE].

[79] S. Davidson, E. Nardi and Y. Nir, Leptogenesis, Phys. Rept. 466 (2008) 105 [arXiv: 0802.2962] [INSPIRE].

[80] E.W. Kolb and M.S. Turner, The Early Universe, Front. Phys. 69 (1990) 1 [inSPIRE].

[81] A. Pilaftsis and T.E.J. Underwood, Resonant leptogenesis, Nucl. Phys. B 692 (2004) 303 [hep-ph/0309342] [INSPIRE].

[82] W. Buchmüller, P. Di Bari and M. Plümacher, The Neutrino mass window for baryogenesis, Nucl. Phys. B 665 (2003) 445 [hep-ph/0302092] [INSPIRE].

[83] A. Abada, S. Davidson, A. Ibarra, F.X. Josse-Michaux, M. Losada and A. Riotto, Flavour Matters in Leptogenesis, JHEP 09 (2006) 010 [hep-ph/0605281] [INSPIRE].

[84] E. Nardi, Y. Nir, E. Roulet and J. Racker, The Importance of flavor in leptogenesis, JHEP 01 (2006) 164 [hep-ph/0601084] [INSPIRE]. 
[85] P.S.B. Dev, P. Di Bari, B. Garbrecht, S. Lavignac, P. Millington and D. Teresi, Flavor effects in leptogenesis, Int. J. Mod. Phys. A 33 (2018) 1842001 [arXiv:1711.02861] [INSPIRE].

[86] P.S.B. Dev, P. Di Bari, B. Garbrecht, S. Lavignac, P. Millington and D. Teresi, Flavor effects in leptogenesis, Int. J. Mod. Phys. A 33 (2018) 1842001 [arXiv:1711.02861] [InSPIRE].

[87] P. Di Bari, Seesaw geometry and leptogenesis, Nucl. Phys. B 727 (2005) 318 [hep-ph/0502082] [INSPIRE].

[88] S. Blanchet and P. Di Bari, Leptogenesis beyond the limit of hierarchical heavy neutrino masses, JCAP 06 (2006) 023 [hep-ph/0603107] [INSPIRE].

[89] G. Engelhard, Y. Grossman, E. Nardi and Y. Nir, The Importance of N2 leptogenesis, Phys. Rev. Lett. 99 (2007) 081802 [hep-ph/0612187] [INSPIRE].

[90] B. Adhikary, M. Chakraborty and A. Ghosal, Masses, mixing angles and phases of general Majorana neutrino mass matrix, JHEP 10 (2013) 043 [Erratum ibid. 09 (2014) 180] [arXiv: 1307.0988] [INSPIRE].

[91] Majorana collaboration, N. Abgrall et al., The Majorana Demonstrator Neutrinoless Double-Beta Decay Experiment, Adv. High Energy Phys. 2014 (2014) 365432 [arXiv: 1308.1633] [INSPIRE].

[92] M. Agostini, G. Benato and J. Detwiler, Discovery probability of next-generation neutrinoless double- $\beta$ decay experiments, Phys. Rev. D 96 (2017) 053001 [arXiv:1705. 02996] [INSPIRE].

[93] C. Hagedorn and E. Molinaro, Flavor and CP symmetries for leptogenesis and $0 \nu \beta \beta$ decay, Nucl. Phys. B 919 (2017) 404 [arXiv:1602.04206] [InSPIRE].

[94] E.K. Akhmedov, M. Frigerio and A.Yu. Smirnov, Probing the seesaw mechanism with neutrino data and leptogenesis, JHEP 09 (2003) 021 [hep-ph/0305322] [INSPIRE].

[95] P. Di Bari, L. Marzola and M. Re Fiorentin, Decrypting SO(10)-inspired leptogenesis, Nucl. Phys. B 893 (2015) 122 [arXiv: 1411.5478] [INSPIRE]. 